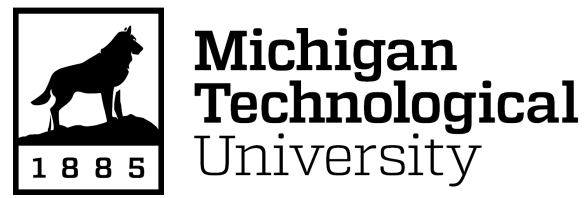

Michigan Technological University Digital Commons @ Michigan Tech

Dissertations, Master's Theses and Master's Reports

2021

\title{
Atomistic continuum simulations for nano-indentation and compression of multi-layer graphene
}

Ashwini Nikumbh

Michigan Technological University, aanikumb@mtu.edu

Copyright 2021 Ashwini Nikumbh

\section{Recommended Citation}

Nikumbh, Ashwini, "Atomistic continuum simulations for nano-indentation and compression of multi-layer graphene", Open Access Master's Thesis, Michigan Technological University, 2021.

https://doi.org/10.37099/mtu.dc.etdr/1165

Follow this and additional works at: https://digitalcommons.mtu.edu/etdr

Part of the Applied Mechanics Commons 
ATOMISTIC CONTINUUM SIMULATIONS FOR NANO-INDENTATION AND COMPRESSION OF MULTI-LAYER GRAPHENE

By

Ashwini Nikumbh

\section{A THESIS}

Submitted in partial fulfillment of the requirements for the degree of MASTER OF SCIENCE

In Mechanical Engineering

MICHIGAN TECHNOLOGICAL UNIVERSITY

2021

(C) 2021 Ashwini Nikumbh 
This thesis has been approved in partial fulfillment of the requirements for the Degree of MASTER OF SCIENCE in Mechanical Engineering.

Department of Mechanical Engineering - Engineering Mechanics

Thesis advisor: Dr. Susanta Ghosh

Committee Member: Dr. Gregory M. Odegard

Committee Member: Dr. Yoke Khin Yap

Department Chair: Dr. William W. Predebon 


\section{Contents}

List of Figures $\quad$ v

Acknowledgement vii

Abstract viii

\begin{tabular}{lll}
\hline & Introduction & 1
\end{tabular}

2 Atomistic Continuum Model 2

2.1 Kinematics . . . . . . . . . . . . . . . . . . . . . . . . 2

2.2 Strain Energy Density . . . . . . . . . . . . . . . . . . . . . . . . . . . 3

2.2 .1 Bonded Interaction Potential . . . . . . . . . . . . . . . . . 3

$2.2 .2 \quad$ Non-Bonded Interaction Potential between Graphene Layers . . . . . . . . . 5

2.2.3 Non-bonded Interaction Potential between Graphene and Substrate . . . . . 6

2.2.4 Non-bonded Interaction Potential between Graphene and Indentor . . . . . . 6

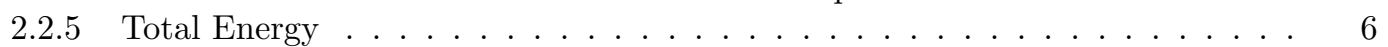

2.3 Numerical Implementation $\ldots \ldots \ldots$. . . . . . . . . . . . . . . . . . . . . . 6

2.4 Equilibrium configuration $\ldots \ldots \ldots \ldots \ldots \ldots \ldots$

2.4 .1 Newton Raphson . . . . . . . . . . . . . . . . . . . . . . . . . . 8

2.4 .2 L-BFGS $\ldots \ldots \ldots \ldots \ldots$

$2.4 .3 \quad$ Internal Energy $\ldots \ldots \ldots \ldots \ldots \ldots$

2.4 .4 Internal Force $\ldots \ldots \ldots \ldots$. . . . . . . . . . . . . . . . . . . . . 9

3 Mathematical models used to represent 2D materials $\quad 11$

3.1 Doubly Clamped Beam Model: . . . . . . . . . . . . . . . . . . . . . . . . . . . 11

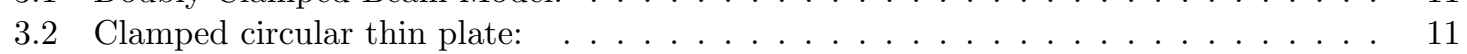

3.3 Sandwich structure under centrally pointed load: . . . . . . . . . . . . . . . . . 11

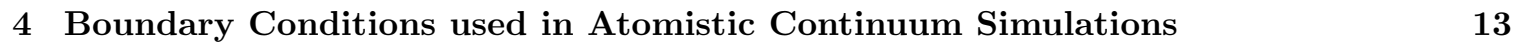

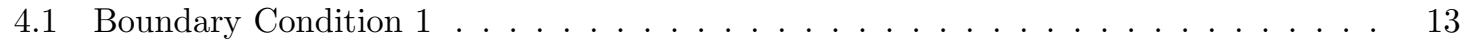

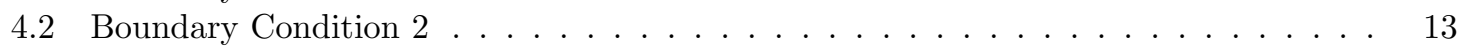

4.3 Boundary Condition $3 \ldots \ldots \ldots \ldots \ldots \ldots \ldots$

5 Measurement of Elastic Properties of Multi-layer Graphene Using Nanoindentation Technique 15

5.1 Nanoindentation Experiment by Aleksey Falin et al. [9] . . . . . . . . . . . . . . 15

5.2 Nanoindentation Experiment by Lee et al. 13$] \ldots \ldots \ldots \ldots$

$\begin{array}{lll}6 & \text { Results and Discussion } & 17\end{array}$

6.1 Atomistic Continuum Simulation Results to Reproduce Nano-indentation Experiment Conducted by Aleksey Falin et al. . . . . . . . . . . . . . . . . . 17

6.1.1 Atomistic continuum simulations for suspended bi-layer graphene . . . . . . 17

6.1.2 Atomistic continuum simulations for suspended tri-layer graphene . . . . . . . 19

6.2 Atomistic Continuum Simulation Results to Reproduce Nano-indentation Experiment Conducted by Lee et al. . . . . . . . . . . . . . . . . . . . . . 21

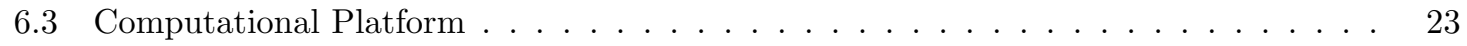

6.3 .1 Nanoindentation Simulation . . . . . . . . . . . . . . . . . . . . 23

6.3 .2 Uniaxial and Biaxial Compression Simulation . . . . . . . . . . . . . . . 23

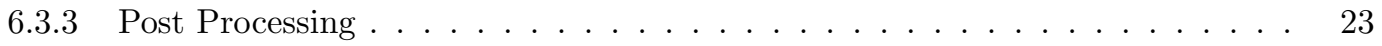


7 Uniaxial and Biaxial Compression of Graphene to Study the Surface Morphology: 24

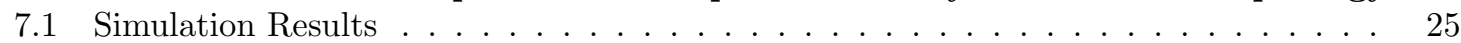

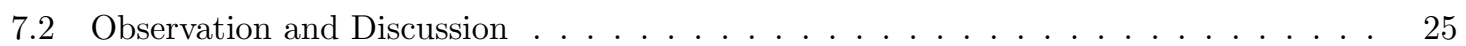

7.3 Results of Uniaxial Compression: . . . . . . . . . . . . . . . . . . . . . 26

7.3.1 Weak Adhesion Potential Between Graphene Layers (0.096 aJ/nm2). . . . . . 26

7.3.2 $\quad$ Strong Adhesion Potential Between Graphene Layers (0.96 aJ/nm2). . . . . . 26

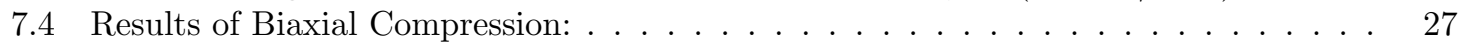

7.4.1 Weak Adhesion Potential Between Graphene Layers (0.096 aJ/nm2). . . . . . 27

7.4.2 $\quad$ Strong Adhesion Potential Between Graphene Layers (0.96 aJ/nm2). . . . . . 27

$\begin{array}{lll}8 & \text { Future Scope } & 29\end{array}$

\begin{tabular}{lll}
\hline & Conclusion & 30
\end{tabular}

\begin{tabular}{ll}
\hline References & 31
\end{tabular}

\begin{tabular}{|l|l|l|l|l}
\hline Copyright Documentation & 33
\end{tabular} 


\section{List of Figures}

\begin{tabular}{|c|c|c|}
\hline & showing the configuration space, deformation maps, and & \\
\hline & coordinate axes [2]. . & 2 \\
\hline 2 & The exponential map representing the transformation of the vector $a=F A$ from & \\
\hline & planer undeformed configuration to planer deformed configuration $a[2] \ldots \ldots$ & \\
\hline 3 & The exponential map representing the transformation of the vector $w=F A$ tangent & \\
\hline & 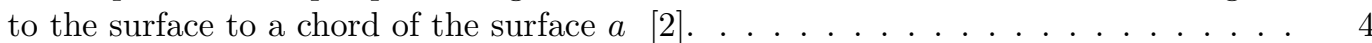 & \\
\hline 4 & The hexagonal cells of graphene in multi-lattice honeycomb arrangement 2 . & 5 \\
\hline 5 & Mapping of parent element to the actual surface $[18][\ldots \ldots \ldots \ldots$ & 7 \\
\hline 6 & Convergence of total energy for 12000 iterations.. . . & \\
\hline 7 & Image of Convergence of norm of residual force for 12000 iterations. & \\
\hline 8 & Schematic of doubly-clamped beam structure for 2D material before and after apply- & \\
\hline & 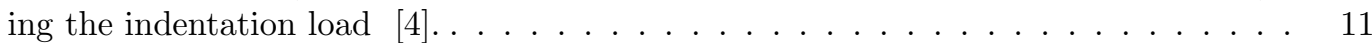 & \\
\hline 9 & Schematic of clamped circular thin plate model for 2D material under indentation & \\
\hline & lod 4 d & \\
\hline 10 & Schematic of sandwich beam geometry under centrally pointed load $[9$. . & \\
\hline 11 & \begin{tabular}{|l|l|} 
Schematic of Boundary condition 1. & $\ldots \ldots \ldots \ldots$
\end{tabular} & \\
\hline 12 & Schematic of Boundary condition 2. & \\
\hline 13 & Schematic of Boundary condition 3 . & \\
\hline 14 & (a) AFM images of multi-layer graphene 9 . (b) Load-displacment data and curve & \\
\hline & fitting of Eq. (35) for monolayer, bilayer and trilayer graphene $9 . .1$. & \\
\hline 15 & Experimental Results for the elastic properties of multilayer graphene [16]. (A) Images & \\
\hline & of suspended graphene membranes. (B) AFM image of monolayer graphene, 1.5 & \\
\hline & $\mathrm{mm}$ in diameter. (C) Image of AFM experimental setup. (D) AFM image of a & \\
\hline & fractured membrane 13 . (E) Load-displacement data and curve fitting of Eqn. (35) & \\
\hline & of suspended bilayer graphene membranes $[13 . \ldots \ldots \ldots \ldots \ldots$ & \\
\hline 16 & Comparison of Load - displacement curves for bi-layer graphene obtained from the & \\
\hline & simulation (Red curve) with the load-displacement curve reported in the experiment & \\
\hline & (blue curve) conducted by Aleksey Falin et al., $[9$ consider zero initial pretension.. & \\
\hline 17 & Post-processed results showing radial and out-of-plane deformation of bi-layer & \\
\hline & graphene for $50 \mathrm{~nm}$ indentation depth. . . . . . . . & \\
\hline 18 & Comparison of Load - displacement curve for tri-layer graphene obtained from the & \\
\hline & simulation (Red curve) with the load-displacement curve reported in the experiment & \\
\hline & (blue curve) conducted by Aleksey Falin et al., $[9]$ consider zero initial pretension. & \\
\hline 19 & Post-processed results showing radial and out-of-plane deformation of tri-layer & \\
\hline & graphene for $50 \mathrm{~nm}$ indentation depth. . . & \\
\hline 20 & Comparison of Load - displacement curve for bi-layer graphene obtained from the & \\
\hline & simulation (Red curve) with the load-displacement curve reported in the experiment & \\
\hline & \begin{tabular}{ll|l} 
(blue curve) conducted by Lee et al., $[13$ & consider zero initial pretension.
\end{tabular} . & \\
\hline 21 & Post-processed results showing radial and out-of-plane deformation of bi-layer & \\
\hline & graphene at $120 \mathrm{~nm}$ indentation depth. $\ldots \ldots \ldots \ldots \ldots \ldots \ldots$ & \\
\hline 22 & a, schematic illustration of deformation of a graphene membrane on a biaxially pre- & \\
\hline & stretched substrate followed by relaxation of the substrate. b-e, SEM images of & \\
\hline & fferent morphologies generated on the graphene sheet: (b) shows wrinkles formation, & \\
\hline & then formation of delaminated buckles formation as the substrate relaxed uniaxially & \\
\hline & (c), followed by the formation of crumples as the substrate is relaxed biaxially (d), & \\
\hline & afolding of graphene as the substrate is biaxially stretched back (e). (f) & \\
\hline & he crumpling of a single-layer graphene under uniaxial & \\
\hline & Mater. 12 (2013) 321-325 [25]. & \\
\hline
\end{tabular}


23 Post processed images of atomistic simulation results showing ripples and wrinkles formation on graphene layers under $5 \%$ uniaxial compression strain and weak adhesion potential. ........................... 26

24 Postprocessed images of atomistic simulation results showing ripples and wrinkles formation on graphene layers under 5\% uniaxial compression strain and strong adhesion. 26

25 Postprocessed images of atomistic simulation results showing ripples and wrinkles formation on graphene layers under 5\% biaxial compression strain and weak adhesion. 27

26 Postprocessed images of atomistic simulation results showing ripples and wrinkles formation on graphene layer under 5\% biaxial compression strain and strong adhesion. 27

27 Ampltitude - compression plots for bilayer graphene subjected to uniaxial and biaxial compression strain at different adhesion potential . . . . . . . . . . . . . . . . . . 28 


\section{Acknowledgement}

First, I would like to thank God, for showering his blessings over me, which I do believe helped me to complete my research successfully.

I would like to thank my advisor, Dr. Susanta Ghosh, for providing me this opportunity to work on this exciting research topic and also providing me with his invaluable guidance throughout this research. His scientific approach, in-depth knowledge about the research topic, and troubleshooting techniques have helped me to a very great extent to accomplish this research. It was a great privilege to work with him.

I would like to thank my thesis defence committee members, Dr. Gregory M. Odegard and Dr. Yoke Khin Yap to be a part of my defence committee and for their suggestions, advise and detailed examination of my work.

I would also like to offer my deep and sincere gratitude to my research colleague Upendra Yadav for making the atomistic-continuum simulation code available to me. His guidance, knowledge, and moreover the willingness to share his knowledge with me, patiently, enabled me to complete this research on time. I also would like to acknowledge and appreciate all the support I received from Revanth Mattey, Shashank Pathrudkar, and Ponkrshnan Thiagarajan thoughout the course of this research.

I would like to thank the Michigan Technological University, The Graduate School and the Department of Mechanical Engineering for providing me with adequate resources and training required for this research work and also for giving me a Grader position which helped me financially during the Masters. I would also like to thank National Science Foundation for funding this code.

I want to thank XSEDE and Superior for sharing their high-performance computing facilities which was critical for this research, J. Robert Van Pelt and John and Ruanne Opie Library for making the library computers available for this work and the Michigan Tech IT services for making the MATLAB and Tecplot 360 License available.

Above all, I am extremely grateful to my parents for proving me with all the support needed to attend a graduate school in the United States of America and for their constant love and care though out my Masters. 


\section{Abstract}

Graphene has attracted a great share of research interest due to its extraordinary electrical, thermal, mechanical, and physical properties. Such spectacular properties of graphene open a wide range potential of applications in electronics, energy storage, composites, and biomedical fields. The mechanical properties of graphene can have a huge impact on its performance in graphene-based devices and thus it is important to study them. But the difficulties in experimental characterization and computational limitations to simulate large graphene sample consisting of billions of atoms makes it a challenging task. Thus, accurate and efficient simulation tools to predict the complex deformation of large graphene samples are needed but are still elusive.

The objective of this thesis is to utilize the atomistic-continuum foliation (AC) model developed by Ghosh and Arroyo (2013) and modified by Upendra Yadav, to reproduce the nanoindentation experiments accurately. This atomistic - continuum foliation (AC) model enables one to directly reproduce the experimental results, something that was not possible before. Using this model we can study the effects of different variables like adhesion, frictional force, indenter radius and stress concentration which is not possible to obtain from the experiments.

This thesis also includes the simulation results for different surface morphology like wrinkles and ripples generated on the multi-layer graphene samples under uniaxial and biaxial compression for different van der Waals potential, which paves a way to study the multifunctionality and control of crumpling and unfolding of graphene to enhance its performance in graphene-based devices. 


\section{Introduction}

Carbon is one of the most abundant element in universe, forming backbone of every living organism on earth. It is capable of forming hard diamond or soft graphite depending upon its structure. Graphene is a 2D crystalline carbon allotrope, formed of a covalently bonded (sp2 - hybridized) single layer of carbon atoms 27. Graphene is the strongest and thinnest material which forms the basis of 3D graphite to 1D carbon nanotubes 27. Since its discovery, graphene has attracted a great share of research interest due to its extraordinary 2D lattice structure and unique electrical, thermal, mechanical and physical properties 25]. Such fascinating properties of graphene has driven a stimulating research for its application in nanotechnology [19], like micro- and nanoelectromechanical systems (MEMS/NEMS) consisting of integrated circuits [14, transparent conducting electrodes [17], single molecule gas detectors [22, transistors [11, optical modulators [16] along with its applications in surface coating, composite and sensing. Other Two-dimensional nanomaterials like boron nitride (h-BN), tungsten disulfide $\left(W S_{2}\right)$, phosphorene and molybdenum disulfide $\left(M o S_{2}\right)$ nanosheets have also generated a worldwide research interest due to their unique properties from insulating h-BN to semi-conducting $M o S_{2}$, promising wide range of application [9], [5, [15, 20].

Amongst many unique properties of graphene, mechanical properties such as Young's modulus $E$, Poisson ratio $\nu$, and fracture strength $\sigma_{f}$ play a crucial role for their performance in 2D nano-devices 1]. Due to which, mechanical properties of graphene have been extensively studied theoretically, experimentally and numerically. Experimental and simulation studies have reported that the surface morphology of graphene and CNT can be controlled through external perturbation like bending, compression, tension, in-plane shear, and thermally induced stresses. Several attempts are made to control the ripple structure formation on the suspended graphene surface for improving their performance in 2D nanodevices [27. These mechanical properties can be effectively studied through nanoindentation and compression loading of free-standing graphene films supported on the soft elastic substrate [27, [1.

A new surge of interest has been generated by stacking layers of graphene on top of one another, which are actively used in high - performance Nano-electromechanical devices and composite design reinforcements 23. Understanding the surface morphologies in multilayer graphene is important as well as challenging due to the difficulties in experimental characterization and atomistic modelling for unknown boundary conditions [23. Thus, investigation of the mechanical properties of such layered structure is essential and studied experimentally and numerically under mechanical loading like compression, tension, bending, stretching and hydrostatic pressure 8$]$.

The objective of this thesis is to compare our recently developed state-of-the-art atomisticcontinuum (AC) models by Ghosh and Arroyo (2013) [24, with the nanoindentation experiments conducted by Aleksey Falin et al. 9] and Lee et al. [13], to estimate the elastic properties of largescale multilayer graphene samples and study its deformation mechanism. Other Numerical methods used to simulate 2D material like Density Functional Theory (DFT), Quantum-mechanics, and Molecular-dynamic simulations are accurate, but they are computationally restrictive to the largescale graphene samples tested experimentally 24]. Besides, our atomistic-continuum (AC) model can consistently simulate large size graphene samples making it possible to directly reproduce the experimental results. Aleksey Falin et al 23, and Lee et al. [13, used Atomic Force Microscopy technique to measure elastic properties of one to three-layered graphene films, the experimental results of which are reproduced in this thesis work. Such comparison has never been reported before because directly reproducing the experimental observation through atomistic simulations has been a major challenge due to the large size of graphene samples tested experimentally 24. Nonlinear elastic behavior is observed in the load-displacement curve for two- and three-layered graphene sheets in both simulation and experiment. Young's modulus is computed by obtaining the slope of the load-displacement curve for both two- and three-layered graphene sheets 23 .

The second part of this thesis includes the large-scale AC simulation results showing multiple morphological deformation phases (wrinkles and ripples) observed in bilayer graphene samples under uniaxial and biaxial compression for different adhesion potential between graphene layers. 


\section{Atomistic Continuum Model}

Atomistic and continuum-based models are the two different computational modeling approaches widely used in the field of material science to understand the mechanical behavior of materials. In atomistic based modeling approach, collective behavior of atoms and its interaction is modeled on large scale, considering the atomic physics. But, this approach is computationally expensive and time consuming. Continuum-based model are used to model materials by considering it as continuous mass, but this approach ignores atomic physics. This thesis introduces the Atomisticcontinuum modeling approach that combines the atomistic and continuum modeling approaches to simulate crystalline materials. Surface [2] model and Foliation 24] model are the two known models developed by this approach.

In the experiments, periodic wavelike deformation patterns can be seen in the supported graphene layer called rippling 24]. Graphene consists of crystalline membrane having very high Young's modulus and very low bending modulus 24]. Besides, the non-bonded interactions also called van der Waals interactions or adhesion potential guide the deformation patterns and keep the graphene layers separated [24]. Under loading, the graphene layers bend to minimize their inplane strain following a near isometric deformation, leading to wrinkling patterns 24]. Accurate and efficient simulation tools to predict the complex deformation of large graphene samples are needed but are still elusive 24]. MD, Quantum-mechanical, and DFT simulations are consistently accurate, but they are computationally expensive to simulate large graphene samples having millions of atoms 24. Besides this, the atomistic-continuum (AC) model combines both atomistic and continuum models, which follows a differential-geometric concept called Foliations [24. The Foliation model uses an inter-layer 4-10 Lennard-Jones potential for van der Waals interactions to describe nonbonded interactions and a modified Brenner potential to describe bonded-interactions 24.

This atomistic-continuum foliation (AC) model can simulate a large-scaled 2D membranes accurately, thus making it best suitable for the simulation of large graphene samples used in experimental testing and real-world applications. It is now possible to directly reproduce the experimental results using this atomistic-continuum foliation (AC) model developed by Ghosh and Arroyo (2013) [10, which is the main focus of this thesis.

\section{$2.1 \quad$ Kinematics}

A configuration mapping function $\left(\varphi_{0}\right)$ maps a body from parametric configuration to the local reference configuration. Another mapping function $(\varphi)$ is defined from parametric configuration to Euclidean configuration, $R^{3}$ as shown in the Figure. 1 [24].

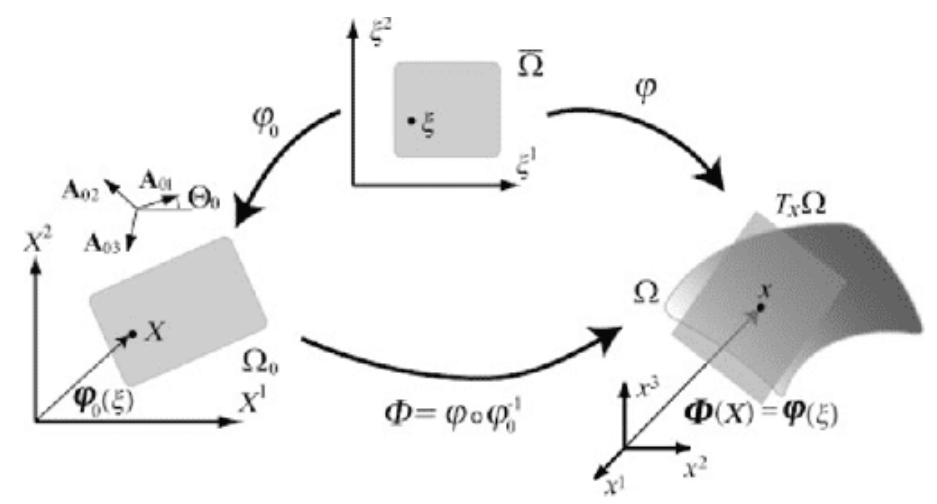

Figure 1: Schematic of kinamatics showing the configuration space, deformation maps, and coordinate axes [2].

The deformation map can then be defined as $\Phi=\varphi \circ \varphi_{0}^{-1}$. Using this deformation map, the 
deformation gradient can be expressed as,

$$
F=D_{\xi} \varphi D_{\xi} \varphi_{0}^{-1}
$$

Using the homogeneous deformation, the stretch and shear free reference configuration can be expressed as

$$
T_{0}(\xi)=\left[\begin{array}{ccc}
2 \pi\left[R_{\text {inn }} \xi^{3}+R_{\text {out }}\left(1-\xi^{3}\right)\right] & 0 & 0 \\
0 & L & 0 \\
0 & 0 & R_{\text {out }}-R_{\text {inn }}
\end{array}\right]
$$

where $R_{\text {inn }}$ and $R_{\text {out }}$ is the inner and outer radius and $\mathrm{L}=$ length of graphene sample. The deformation gradiant can be expressed as,

$$
F=D_{\xi} \varphi T_{0}^{-1}
$$

The Cauchy-Green strain tensor of the layers can be expressed as,

$$
C^{l}=\left(T_{0}^{l}\right)^{-T} g\left(T_{0}^{l}\right)^{-1}
$$

where $g=\frac{\partial \varphi}{\partial \xi^{\alpha}}$ is the metric tensor of each layer at each point. To obtain curvature tensor, the pull-back of the Weingertan map can be computed as,

$$
K=\left(T_{0}^{l}\right)^{-T} k\left(T_{0}^{l}\right)^{-1}
$$

where $K$ is the curvature of graphene layer, obtained as component of Weingertan map and can be expressed as $K_{\alpha \beta}=<n, g_{\alpha \beta}>$ where, $n$ is the unit normal vector at that point and comma denoted the partial derivative with respect to $\xi^{\alpha}$ [24].

Using exponential Cauchy-Born rule [10, the energy density of the deformed graphene layers can be obtained in terms of right Cauchy-Green's strain field $C^{l}$, the transverse-to-the wall stretch field of the deformation map $(\Phi)$ and the curvature tensor field $K$ can be expressed as $W\left(C^{l}, K\right)$ [24. The formulation of exponential Cauchy-Born rule is explained in detail in the next section.

\section{$2.2 \quad$ Strain Energy Density}

\subsubsection{Bonded Interaction Potential}

Using the Cauchy-Born rule, we can obtain the deformed bond length and bond angles as a function of the continuum variables. According to the standard Cauchy-Born rule, deformation of the lattice vectors can be represented in the form of linear transformation $\mathbf{a}=\mathbf{F}(\mathbf{X}) \mathbf{A}$, where A represents an undeformed vector originating from the reference coordinate $X$ and $a$ represents the deformed vector 2.

where, $\mathrm{F}$ is Deformation Gradient, using Cauchy Born Rule, deformed bondlength and bond angles can be expressed as, $a=F A$ and $\cos \theta=\frac{A \cdot C B}{\|a\| .|| b \|}$ respectively.

The standard Cauchy-Born rule fails to capture the mechanics of curved membrane. Therefore to overcome this limitation, an extension of the Cauchy-Born rule was proposed to take into account the mechanics of curvature of the crystalline membrane [2].

The exponential Cauchy-Born rule can be expressed as,

$$
a=\exp \circ F(X) A
$$




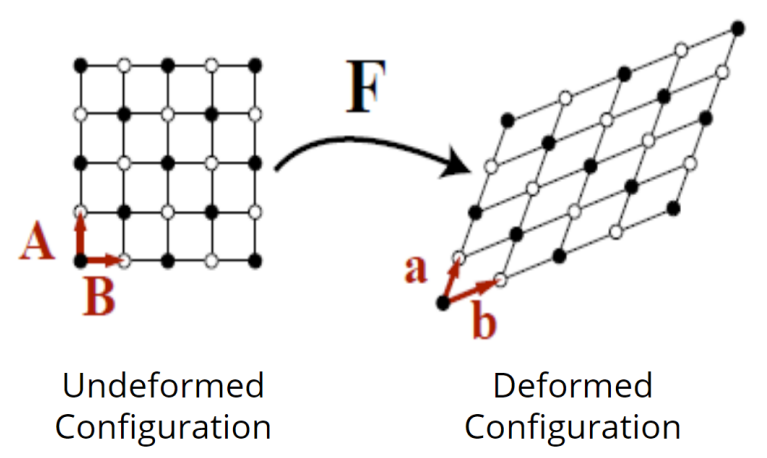

Figure 2: The exponential map representing the transformation of the vector $a=F A$ from planer undeformed configuration to planer deformed configuration $a$ [2]

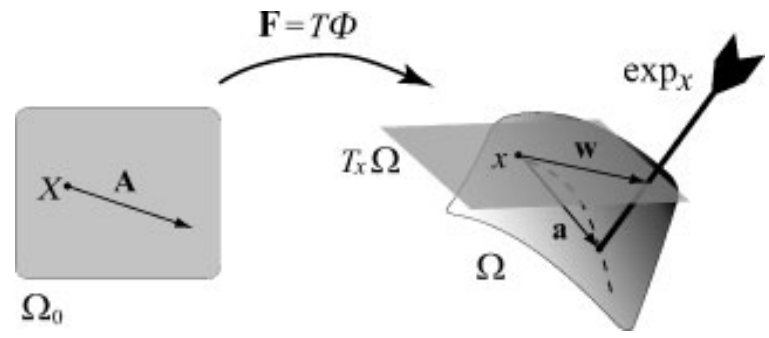

Figure 3: The exponential map representing the transformation of the vector $w=F A$ tangent to the surface to a chord of the surface $a$ [2].

where, exp represents the exponential map of the surface at $\Phi(X)$ [2].

For planer underformed membrane, the exponential Cauchy-Born rule can be expressed as,

$$
a=\exp _{\Phi(X)} \circ F(X) A
$$

The length of a deformed bond and the angle between two deformed bonds a and $\mathrm{b}$ can be estimated by a simple formula,

$$
\begin{array}{r}
a=|\boldsymbol{a}|=\sqrt{a^{c} a^{c}} \\
\text { and } \\
\theta=\arccos \frac{\langle a / b\rangle}{a b}=\arccos \frac{a^{c} b^{c}}{a b}
\end{array}
$$

The bond lengths and the angles can now be expressed as a function of continuum variables $C$ (Cauchy-Green strain tensor) and $K$ (Curvature tensor). We have computed the derived strain measures $a=f(\mathbf{C}, \mathbf{K} ; \mathbf{A})$ and $\theta=g(\mathbf{C}, \mathbf{K} ; \mathbf{A}, \mathbf{B})$ 2. These continnum variables are sufficient to derive the continuum models from the atomistic description of the membrane system considering both lattice structure and inter atomic potential [2], see Figure 3.

Consider Figure 4, it shows the representative hexagonal cell for the graphene honeycomb lattice, containing two nuclei with surface area $S_{0}=(3 \sqrt{3} / 2) A_{0}^{2}$. The strain energy per unit area of the continuum membrane is expressed in the form of Tersoff-Brenner potential [2],

$$
W=W(\boldsymbol{C}, K)=\frac{1}{S_{0}} \sum_{i=1}^{3}\left[V_{R}\left(a_{i}\right)-\vec{B}\left(a_{j}, a_{k}, \theta_{j}, \theta_{k}\right) V_{A}\left(a_{i}\right)\right]
$$




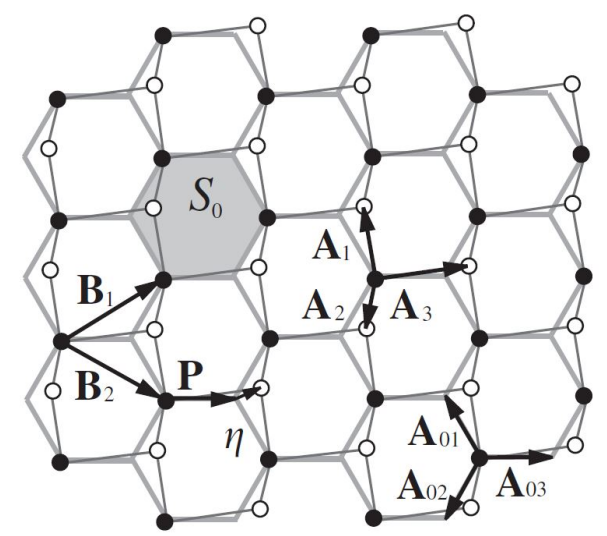

Figure 4: The hexagonal cells of graphene in multi-lattice honeycomb arrangement 2].

The hyper-elastic potential $W$ is systematically derived from the atomistic interactions describing the force and bond energy using a kinematic rule linking continuum and lattice deformations, averaging the atomistic energy in one-unit cell of the lattice, and the exponential Cauchy-Born rule 2. For bonded interaction, the strain energy of graphene membrane can finally be expressed as [24],

$$
\Pi_{1}(\varphi)=W\left(C_{l}, K\right)
$$

A much simpler continuum model called Saint Venant-Kirchhof was used by Arroyo and Belytschko (2005) 2, which provided reasonably accurate results as compared to atomistic continuum simulations. This model is based on the Saint Venant-Kirchhof strain energy density and on the Helfrich curvature energy

$$
W_{K-S V-H}\left(\boldsymbol{C}^{l}, K\right)=\frac{1}{2}\left[2 \mu E^{l}: E^{l}+\lambda\left(\operatorname{tr} \boldsymbol{E}^{l}\right)^{2}\right]+\frac{1}{2}\left[c_{a}(2 H)^{2}+c_{b} K\right]
$$

where $\kappa$ is the mean curvature and $K=\operatorname{det}\left[\left(\boldsymbol{C}^{l}\right)^{-1} K\right]$ is the gaussian curvature. $\mu$ and $\lambda$ are the two-dimensional Lame coefficients [2], $\boldsymbol{E}^{l}=\frac{1}{2}\left(\boldsymbol{C}^{l}-\boldsymbol{I} \boldsymbol{d}\right), \boldsymbol{H}=\frac{1}{2}\left(\boldsymbol{C}^{l}\right)^{-1}, c_{a}$ and $c_{b}$ are the bending elastic modulus. Saint Venant-Kirchhof model is inaccurate in modelling the non-linear system and cannot be used for the large scale simulation of 2D material [10].

\subsubsection{Non-Bonded Interaction Potential between Graphene Layers}

To model the adhesion interaction between graphene layers, a simple pairwise 6-12 Lennard Jones potential is computed as [27]:

$$
V(r)=4 \epsilon\left(-\left(\frac{\sigma}{r}\right)^{6}+\left(\frac{\sigma}{r}\right)^{12}\right)
$$

where, $\mathrm{r}$ denotes distance between two interacting atoms, $\epsilon$ denotes well depth and $\sigma$ denotes equilibrium spacing or distance at which atom-atom potential energy is zero. The above expression estimates van der Waals interaction between single pair of atoms in the system at a time, which makes it infeasible to compute the non-bonded interactions between millions pairs of atoms in a system at a time. Besides, the van der Waals interactions over all atoms pairs in the system can be obtained as a function of the distance between two layers of graphene by considering the sum of pairwise 4-10 Lennard-Jones (LJ) potential expressed as [24,

$$
V_{v d W}=-\frac{\left|V_{g}\left(t_{0}\right)\right|}{0.6}\left[\left(\frac{t_{0}}{t}\right)^{4}-0.4\left(\frac{t_{0}}{t}\right)^{10}\right]
$$


where $V_{g}\left(t_{0}\right)$ is the well-depth and $t_{0}$ is the equilibrium spacing. For mutilayer graphene system, the total volumetric effective local van der Waals interaction can be expressed as [24,

$$
\Pi_{2}(\varphi)=V\left(\lambda^{t}, C^{l}\right)=\frac{N_{w}-1}{N_{w}} \frac{1}{t_{0} J} V_{v d w}\left(\lambda^{t} t_{0}\right)
$$

where $N_{w}$ is the number of layers in a multilayer graphene system, $\lambda^{t}$ is the transversal stretch and $\mathrm{J}$ represents determinant of jacobian of layer.

\subsubsection{Non-bonded Interaction Potential between Graphene and Substrate}

To model the van der Waals interaction between graphene and the substrate, we again use 4-10 Lennard Jones potential [24]. The pair-wise Lennard-Jones 4-10 potential between a graphene and substrate layer can be expressed as,

$$
\Pi_{3}(\varphi)=V_{v d W}=-\frac{\left|V_{g}\left(t_{0}\right)\right|}{0.6}\left[\left(\frac{t_{0}}{t}\right)^{4}-0.4\left(\frac{t_{0}}{t}\right)^{10}\right]
$$

\subsubsection{Non-bonded Interaction Potential between Graphene and Indentor}

The van der Waals intercation between graphene and indentor atoms can be computed by using pairwise 6-12 Lennard Jones potential [27],

$$
\Pi_{4}(\varphi)=V(r)=4 \epsilon\left(-\left(\frac{\sigma}{r}\right)^{6}+\left(\frac{\sigma}{r}\right)^{12}\right)
$$

\subsubsection{Total Energy}

Total internal energy of the system will be the sum of bonded and non-bonded interaction energies expressed as,

$$
\Pi_{i n t}=\int_{\vec{V}}\left(\Pi_{1}(\varphi)+\Pi_{2}(\varphi)+\Pi_{3}(\varphi)+\Pi_{4}(\varphi)\right) d V_{0}
$$

Considering the external energy potential $\pi_{\text {ext }}(\varphi)$ corresponding to the applied external loading, the total energy can be expressed as,

$$
\Pi_{\text {total }}(\varphi)=\Pi_{\text {int }}(\varphi)-\Pi_{\text {ext }}(\varphi)
$$

For different external loading conditions, the deformed shape is obtained by minimization of the total energy w.r.t the deformation map.

\subsection{Numerical Implementation}

A new discretization method called Iso-geometric analysis is used to discritize graphene surface in atomistic continuum model. In this method, basis functions derived from B-Splines approximation are used to generate the deformed shapes exactly. Discritized geometry is obtained by partitioning the parametric body and then mapping the B-spline basis function and control points in parametric space. In classical Iso-parametric analysis (used in Finite Element Method), a nice parent element defined in local coordinate system is mapped into number of distorted elements on a real surface in global coordinate system. Such approximation of geometry leads to errors, like inter-element continuity and discontinuity of higher order derivatives. In Iso-geometric analysis, these errors are eliminated as the entire surface is created by using B-spline basis function, knot vectors and control 


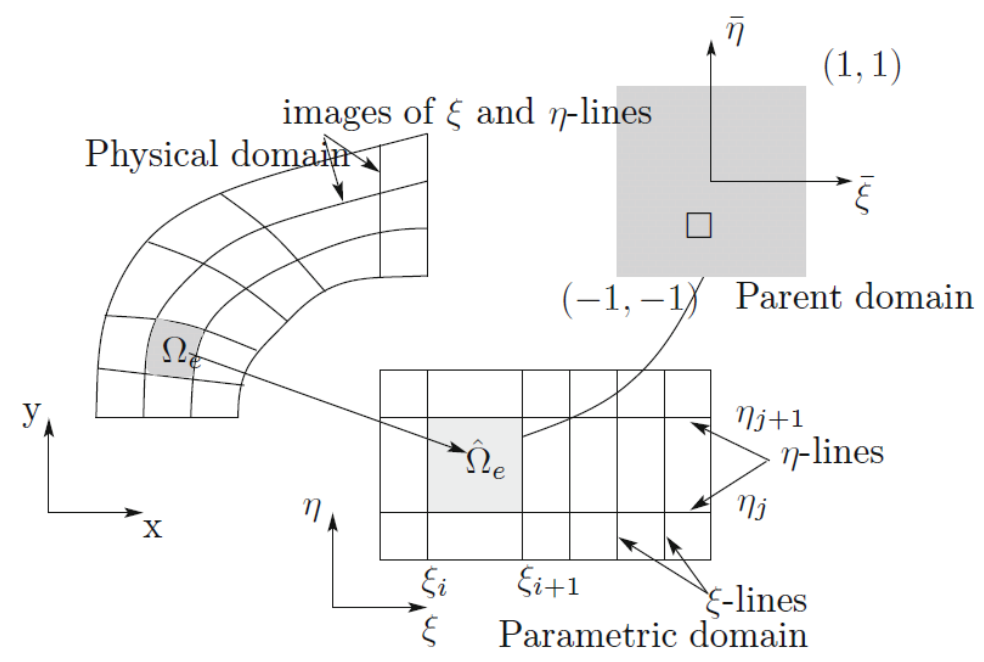

Figure 5: Mapping of parent element to the actual surface [18]

points without any approximation of geometry, see e.g piegl and Tiller (1997).

B-spline basis functions can be starting with piece wise constants $(\mathrm{p}=0)$,

$$
N_{i, 0}=\left\{\begin{array}{rc}
1, & \text { if } \xi_{i}<n<\xi_{i+1} \\
0, & \text { Otherwise }
\end{array}\right.
$$

For $\mathrm{p}=1,2,3, \ldots$., they are defined by,

$$
N_{p, i}(\xi)=\frac{\xi-\xi_{i}}{\xi_{i}+p-\xi_{i}} N_{i, p-1}(\xi)+\frac{\xi_{i}+p+1-\xi}{\xi_{i}+p+1-\xi_{i}+1} N_{i+1, p-1}(\xi)
$$

A knot vector is a set of coordinates in the parametric space, written as $\xi=\xi_{1}, \xi_{2}, \ldots, \xi_{n}+p+1$, where, $\mathrm{i}$ is the knot index, $(\mathrm{i}=1,2, \ldots, \mathrm{n}+\mathrm{p}+1)$, $\mathrm{p}$ is the polynomial order, and $\mathrm{n}$ is the number of basis functions, see piegl and Tiller (1997). In one dimension, basis functions derived from open knot vectors are interpolatory at the ends of the parametric space interval $\left[\xi_{1}, \xi_{n}+p+1\right]$, and at the corners of patches, however they are not interpolatory at the interior knots. This is a differentiating feature between knots and nodes of finite element analysis.

Now consider the following notation, let $\left(\varphi_{0}^{h}\right)_{J}$ be the nodal coefficient in global coordinate system and $\left(\varphi_{0}^{e}\right)_{I}$ be the nodal coefficients in local coordinate system 2. A parametric element $\Omega_{0}^{e}$ is mapped from undeformed space to deformed space through a deformation map $\varphi_{0}^{h}[2]$. A B-spline approximation of the configuration map can be expressed as,

$$
\varphi^{h}\left(\xi_{1}, \xi_{2}, \epsilon_{3}\right)=\sum_{I} \sum_{J} \sum_{K} N_{I}\left(\xi_{1}\right) N_{J}\left(\xi_{2}\right) N_{K}\left(\xi_{3}\right) X_{I J K}
$$

where $N_{I}, N_{J}$, and $N_{K}$ represents the B-spline basis functions and $X_{I J K}$ represents the corresponding control points defining the configuration 24]. Following this discretized configuration map for strain fields can be obtained [24]. A direct minimization of the potential energy $\Pi\left(\varphi^{h}\right)$ can be obtained to accomplish a stable configuration of the discrete system. The numerical methods, namely Newton Raphson and L-BFGS only require gradients of discrete energy w.r.t degree of freedom and can be used effeciently to numerically calculate internal strain energy and non-bonded energy. Detailed explanation of methods used to obtain equilibrium configuration of the system is given in next section. 


\subsection{Equilibrium configuration}

\subsubsection{Newton Raphson}

In numerical optimization, Newton's method is a most iterative method that uses both first and second order derivatives. At a given initial point, Newton Raphson method constructs a quadratic approximation of the objective function that matches first and second order derivatives at that point [6]. We then obtain minimization of the approximate function and uses its minimized value as a starting point in the next step and repeat this process iteratively until we reach minima 3]. In Newton Raphson Method we find the quadratic approximation to the twice (C2) continuously differentiable function $f: R^{n} \rightarrow R$, by obtaining roots of a differentiable function $f$ [6], Considering Taylor's expansion of $f$ about the current point $\mathbf{x}^{k}[6]$,

$$
f(x)=f\left(\boldsymbol{x}^{\boldsymbol{k}}\right)+\left(\boldsymbol{x}-\boldsymbol{x}^{(k)}\right)^{T} \boldsymbol{g}^{(\boldsymbol{k})}+\frac{1}{2}\left(\boldsymbol{x}-\boldsymbol{x}^{(k)}\right)^{T} F\left(\boldsymbol{x}^{(k)}\right)\left(\boldsymbol{x}-\boldsymbol{x}^{(k)}\right) \triangleq q(\boldsymbol{x})
$$

where $g^{(k)}=\nabla F\left(\boldsymbol{x}^{(k)}\right)$, using First order necessary condition to q we get,

$$
\nabla q(\boldsymbol{x})=\boldsymbol{g}^{(k)}+F\left(\boldsymbol{x}^{k}\right)\left(\boldsymbol{x}-\boldsymbol{x}^{(k)}\right)
$$

If $f\left(\boldsymbol{x}^{(k)}\right)>0$ then achieves a minimum at,

$$
\begin{gathered}
\boldsymbol{x}^{(k+1)}=\boldsymbol{x}^{k}-F\left(\boldsymbol{x}^{(k)}\right)^{-1} \boldsymbol{g}^{(k)} \\
0=q^{\prime}(\boldsymbol{x})=\boldsymbol{f}^{\prime}\left(\boldsymbol{x}^{(k)}\right)+\boldsymbol{f}^{\prime \prime}\left(\boldsymbol{x}^{(k)}\right)\left(\boldsymbol{x}-\boldsymbol{x}^{(k)}\right) \\
x^{(k+1)}=x^{(k)}-\frac{f^{\prime}\left(x^{(k)}\right)}{f^{\prime \prime}\left(x^{k}\right)}
\end{gathered}
$$

\subsubsection{L-BFGS}

The Limited-memory Broyden-Fletcher-Goldfarb-Shanno (L-BFGS) algorithm is a limited-memory form of BFGS that is particularly suitable for solving high-dimensional minimization problems [7]. This method belongs to the category of quasi-Newton optimization.

This algorithm obtains solution to the minimization problem by interactively approximating Hessian matrix of the objective function. Using a quadratic approximation, quasi-newton methods locally model function $f\left(x_{k}\right)$ at the point $\overrightarrow{x_{k}}$ at each iteration [7],

$$
Q\left(\overrightarrow{x_{k}}\right)=f\left(\overrightarrow{x_{k}}\right)+\left(\vec{x}-\overrightarrow{x_{k}}\right)^{T} \overrightarrow{g_{k}}+\frac{1}{2}\left(\vec{x}-\overrightarrow{x_{k}}\right) B_{k}\left(\vec{x}-\overrightarrow{x_{k}}\right)
$$

A search direction can then be obtained by computing the vector $\overrightarrow{x *}$ that minimizes $Q(\vec{x})$ [7. Assuming that the Hessian is positive-definite, this is $\overrightarrow{x *}=\overrightarrow{x_{k}}-H_{k} \overrightarrow{g_{k}}$ [7]. The next search point is then found along the ray defined by $\overrightarrow{x_{k}}-\alpha H_{k} \overrightarrow{g_{k}}$. With some degree of convergence tolerance, this process is iterated until we obtain zero [7]. The L-BFGS algorithm generalize well for higher dimensional optimization problems because it avoids storing the sequential approximation of the Hessian matrix. This indeed reduces the cost of memory storage [7. Instead, L-BFGS stores curvature information from the last iterations, more specifically the information about the spatial displacement and the change in gradient, and uses them to find the new search direction without storing or computing the Hessian explicitly [7. Due to the advantage of less memory space requirement and elimination of double derivation step of Newton Raphson, L-BFGS optimization method is used in the atomistic-continuum model for direct minimization of potential energy in multi-layered graphene system [7]. 


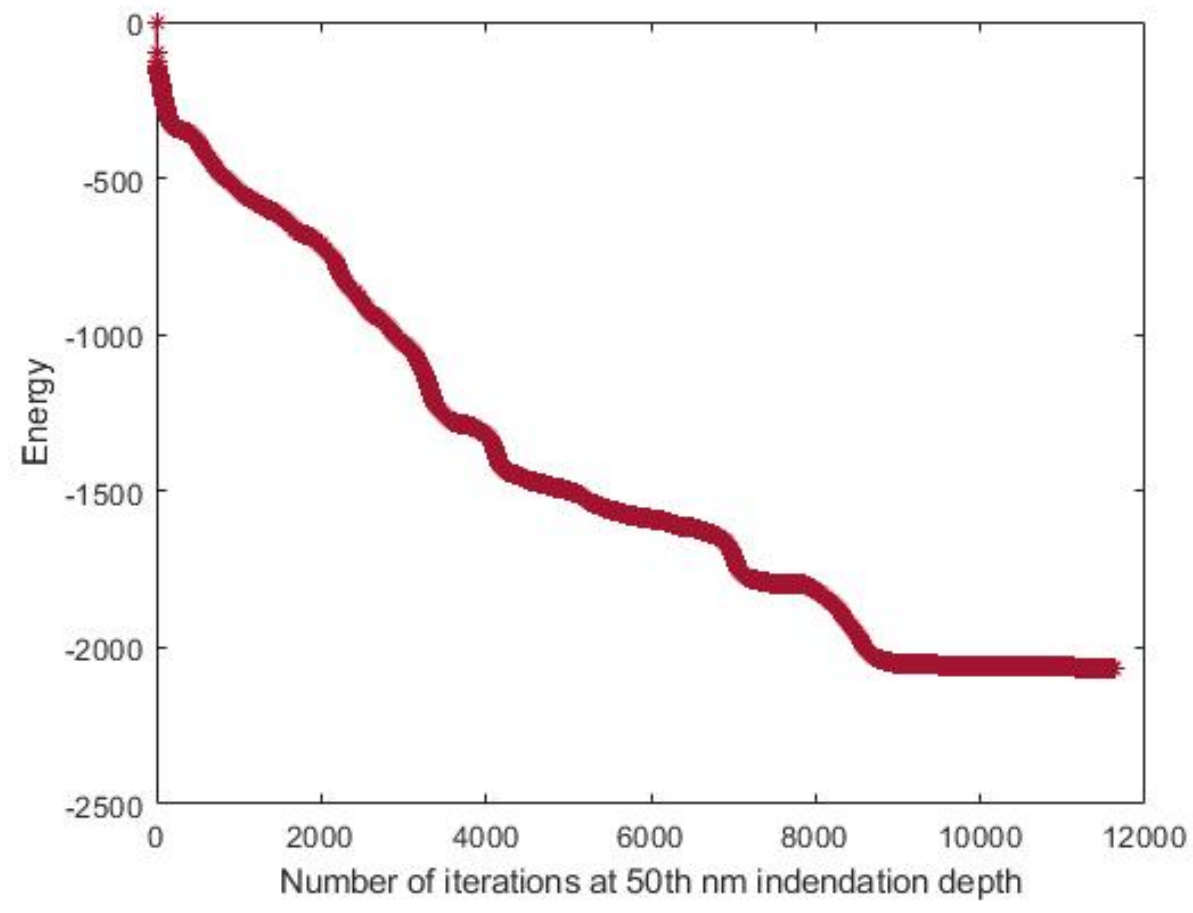

Figure 6: Convergence of total energy for 12000 iterations.

\subsubsection{Internal Energy}

The undeformed configuration $\varphi_{0}^{h}$ is fixed, the deformation map $\Phi^{h}$ is determined by the nodal coefficients $\varphi_{J}^{h}$ [2]. The internal energy for this deformation map is estimated by splitting the integral over the undeformed body into elements and transforming these element integrals to integrals ovre parent element [2]. These integrals are then approximated by numerical Gauss quadrature, expressed as [2],

$$
\Pi_{i n t}\left[\Phi^{h}\right]=\int_{\Omega_{0}} \vec{W}\left(\boldsymbol{C}\left(\Phi^{h}\right), \boldsymbol{K}\left(\Phi^{h}\right)\right) d \Omega_{0}
$$

using the numerical Gauss quadrature,

$$
\Pi_{i n t}\left[\Phi^{h}\right]=\left.\left.\sum_{e=1}^{n e l} \sum_{i=1}^{n i n t} \vec{W}(\boldsymbol{C}, \boldsymbol{K})\right|_{\xi_{i}} \operatorname{det}\left[\left(T \varphi_{0}^{e}\right)_{\alpha}^{A}\right]\right|_{\xi_{i}} \vec{w}_{i}
$$

where $\vec{w}_{i}$ represent the corresponding weights, $\xi_{i}$ represents the quadrature points and nint represents the number of quadrature points for the internal energy [2].

\subsubsection{Internal Force}

The internal forces at nodes of the discretized system can be computed by obtaining the derivatives of the internal energy w.r.t the nodal degrees of freedom [2].

$$
\left(\boldsymbol{f}_{i n t}\right)_{J}=\frac{\partial \boldsymbol{\Pi}_{i n t}}{\partial \varphi_{J}^{h}}
$$



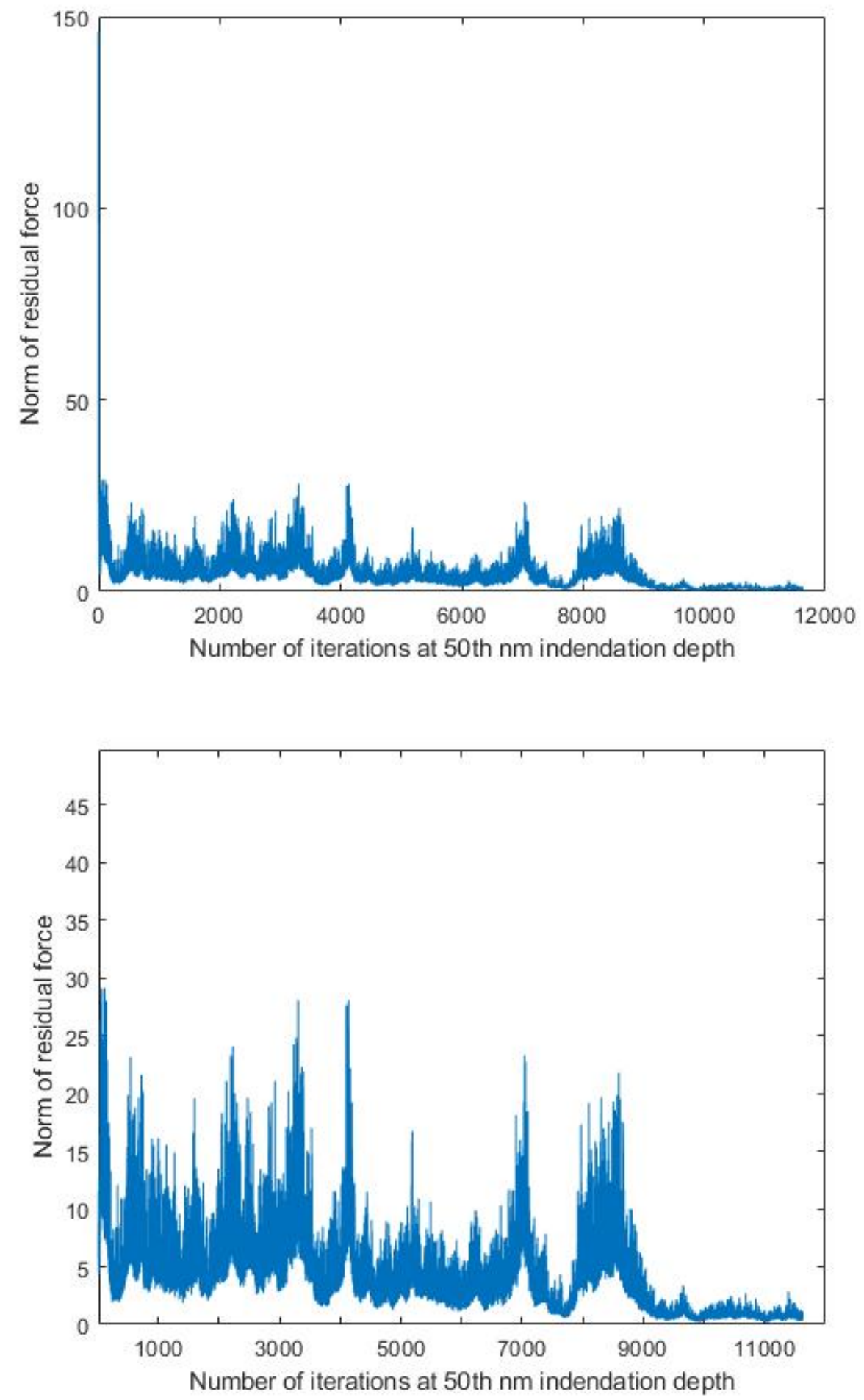

Figure 7: Image of Convergence of norm of residual force for 12000 iterations. 


\section{Mathematical models used to represent 2D materials}

The classical models of beam bending and membrane stretching have been used in the experiments and simulations to predict the indentation response of $2 \mathrm{D}$ materials [4].

\subsection{Doubly Clamped Beam Model:}

Consider a doubly clamped beam section, subjected to a point load at the center of the beam. Relationship between the indentation load and displacement is given by [4]:

$$
P=\left(\frac{\pi^{4} E W}{6}\left(\frac{t}{L}\right)^{3}+\frac{\pi^{2} T}{2 L}\right) \delta_{t}+\frac{\pi^{4} E W t}{8}\left(\frac{\delta_{t}}{L}\right)^{3}
$$

Where, $\mathrm{T}$ is pre-tension in the beam, $\mathrm{W}$ is beam width, $\mathrm{L}$ is beam length and $\mathrm{t}$ is beam thickness.
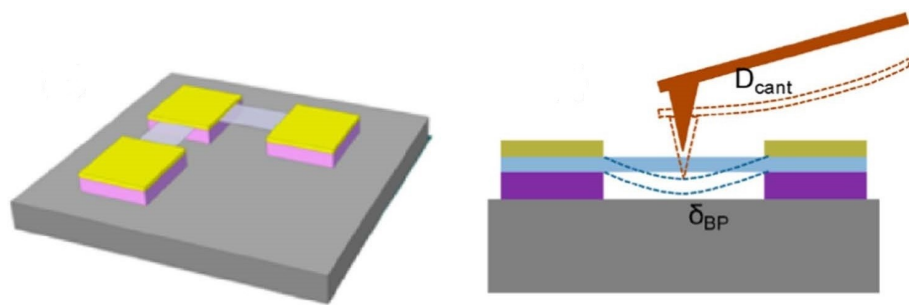

Figure 8: Schematic of doubly-clamped beam structure for 2D material before and after applying the indentation load [4].

\subsection{Clamped circular thin plate:}

Consider a clamped circular thin plate, subjected to a point load at the center of the beam. The relationship between the indentation load and displacement of clamped circular thin plate is given as follows [4:

$$
P=\left(\frac{4 \pi E t^{3}}{3\left(1-\nu^{2}\right) a}+T\right) \frac{\delta_{t}}{a}+E\left(q^{3} t a\right)\left(\frac{\delta_{t}}{a}\right)^{3}
$$

Where, $\mathrm{q}=1 /\left(1.049-0.15 \nu-0.16 \nu^{2}\right)$ is a function of Poisson ratio $\nu, \mathrm{T}$ is pre-tension in the beam, $\mathrm{W}$ is beam width, $\mathrm{L}$ is beam length and $\mathrm{t}$ is beam thickness.

\subsection{Sandwich structure under centrally pointed load:}

Aleksey Falin et al 9], proposed sandwich beam geometries to explain the sliding tendencies in bi-layer graphene shown in the Figure 10. The beam is under a central indentation load F'. F' was set to the measured fracture load proportional to the fractional area of the sandwich beam used. 


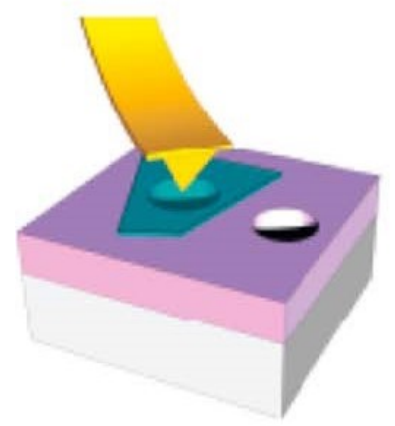

Figure 9: Schematic of clamped circular thin plate model for 2D material under indentation load [4.

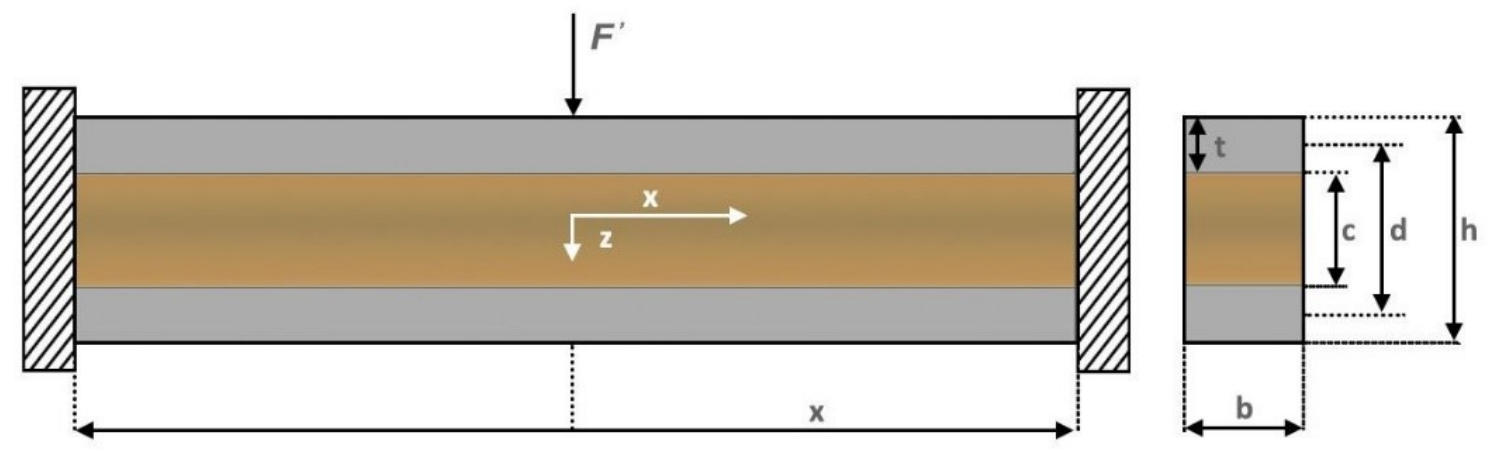

Figure 10: Schematic of sandwich beam geometry under centrally pointed load 9 . 


\section{Boundary Conditions used in Atomistic Continuum Simu- lations}

\subsection{Boundary Condition 1}

In this boundary condition, all graphene layers are fixed outside the substrate hole radius, indenter radius region is constrained in $\mathrm{x}$ and $\mathrm{y}$ direction and allowed to move freely in negative $\mathrm{z}$ direction.

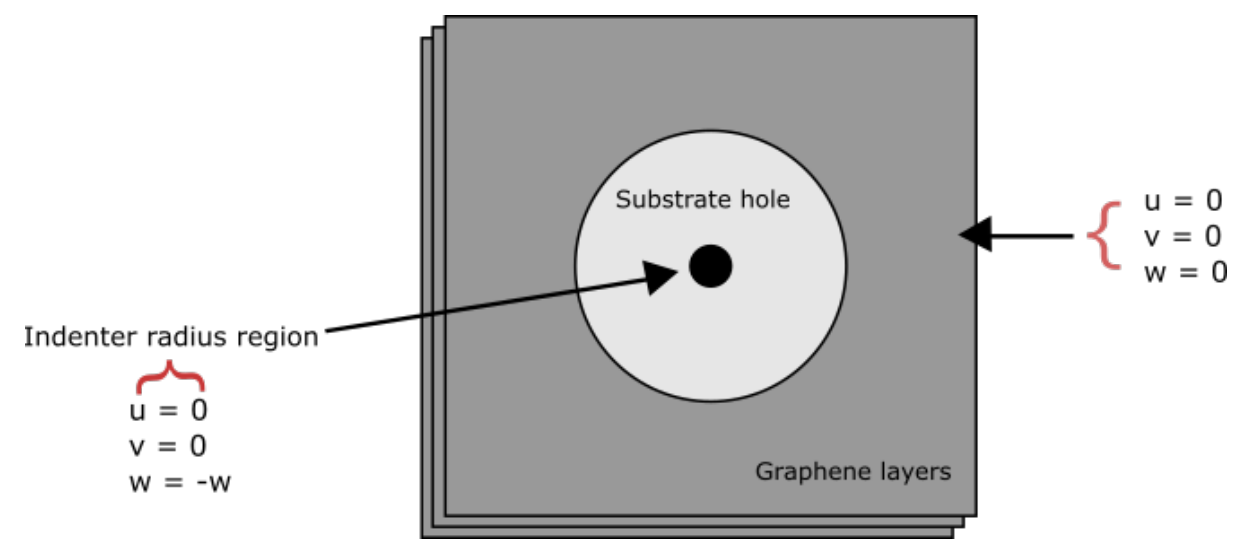

Figure 11: Schematic of Boundary condition 1.

where, $\mathrm{u}, \mathrm{v}$ and $\mathrm{w}$ are the displacements in $\mathrm{x}, \mathrm{y}$ and $\mathrm{z}$ directions respectively.

\subsection{Boundary Condition 2}

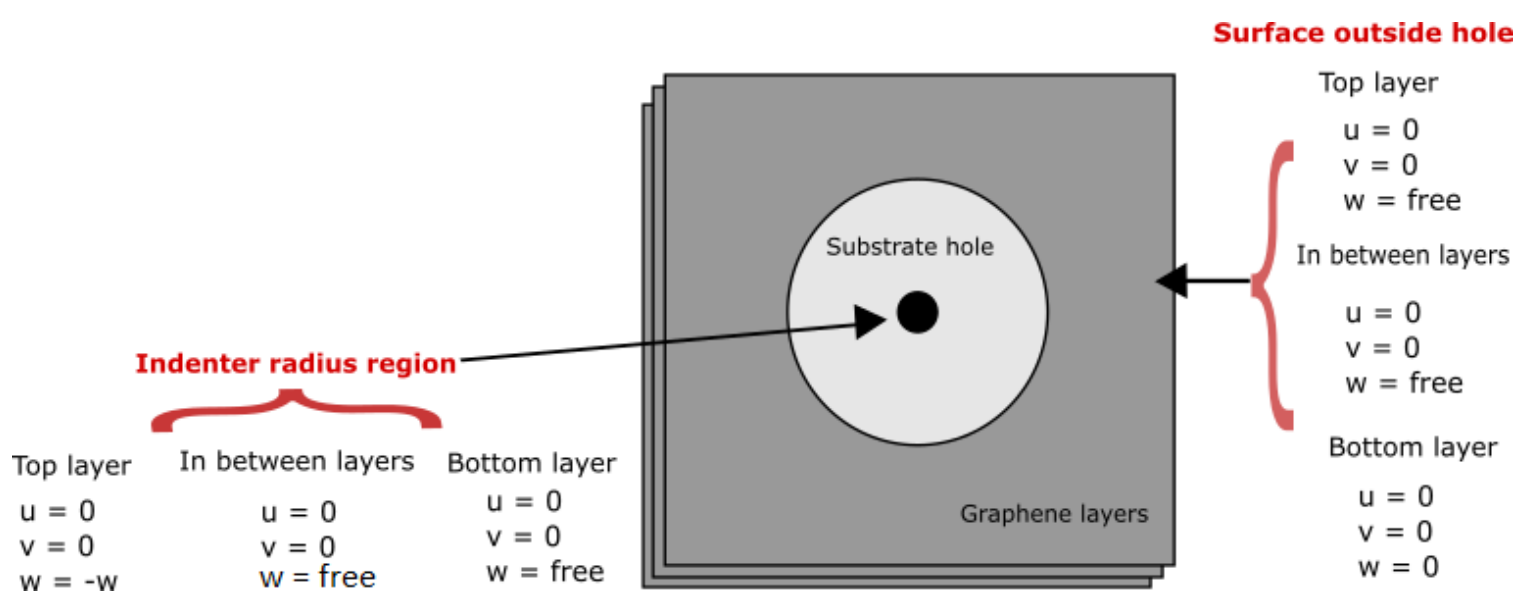

Figure 12: Schematic of Boundary condition 2. 


\subsection{Boundary Condition 3}

Boundary Condition-3 is same as boundary condition-2 except nodal displacement at all edges are fixed in z-direction.

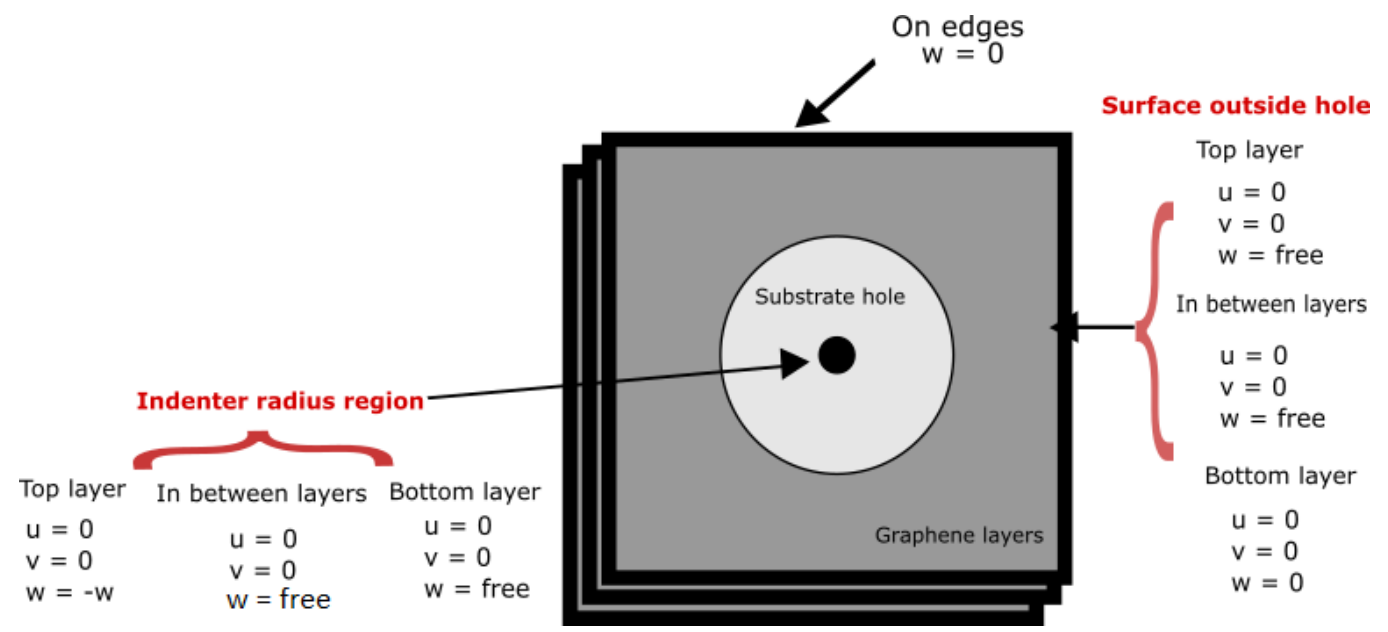

Figure 13: Schematic of Boundary condition 3.

The boundary conditions in the experiments are unknown therefore we ran our simulations using all these three boundary conditions and obtained a good match with the experiment using boundary condition-1, see Figure 11. 


\section{Measurement of Elastic Properties of Multi-layer Graphene Using Nano-indentation Technique}

\subsection{Nanoindentation Experiment by Aleksey Falin et al. [9]}

The extraordinary Young's moduli and third-order elasticity moduli of suspended free-standing graphene sheets are experimentally measured using Atomic force microscopy (AFM) by Aleksey Falin et al 9]. The graphene nanosheets were mechanically exfoliated on a $90 \mathrm{~nm}$ thick silicon oxide-covered silicon $\left(\mathrm{SiO}_{2} / \mathrm{Si}\right)$ substrate with prefabricated micro-wells of $650 \mathrm{~nm}$ in radius [9]. Figure 14. (a) shows atomic force microscopy (AFM) images of one, two- and three-layers graphene lying on the substrate. Two cantilevers with diamond tips of radii $5.6 \mathrm{~nm}$ and $6.3 \mathrm{~nm}$ were used for indentation purposes 9]. The load-displacement curve of multi-layered graphene was fitted till 50 $\mathrm{nm}$ of deflection for estimation of Youngs's modulus [9].

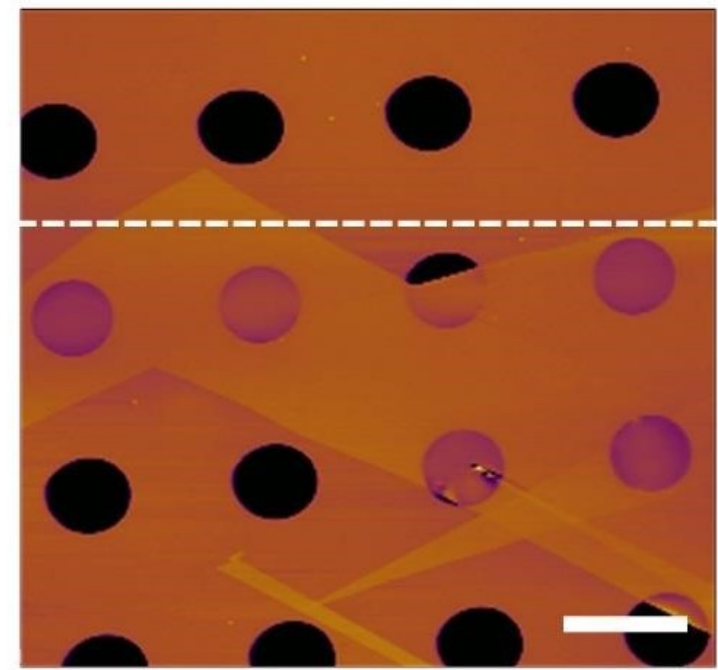

Figure 14: (a) AFM images of multi-layer graphene 9]. (b) Load-displacment data and curve fitting of Eq. (35) for monolayer, bilayer and trilayer graphene [9].

The force-displacement behavior in indentation is interpreted as a result of the nonlinear elastic properties of graphene as shown in Figure 14 (b). Aleksey Falin et al. employed AFM to implement nano-indentation tests and reported experimental values of 2D Young's Modulus for 1, 2 and 3 layers of graphene as $342 \pm 8 \mathrm{Nm}^{-1}, 645 \pm 16 \mathrm{Nm}^{-1}$ and $985 \pm 10 \mathrm{Nm}^{-1}$ [9].

\subsection{Nanoindentation Experiment by Lee et al. [13]}

Lee et al. reported direct measurement of elastic properties and frictional behavior of monolayer and multilayer graphene membranes by implementing the nanoindentation experiment using AFM technique 13. the graphene nanosheets were mechanically exfoliated onto a Si substrate with 300 $\mathrm{nm} \mathrm{SiO} 2$ epilayer patterned with an array of circular wells of diameter 1.5 and $1 \mu \mathrm{m}$ and $500 \mathrm{~nm}$ depth. The thickness of the graphene samples was measured using Raman spectroscopy and AFM contact mode imaging. Optical microscopy was used to identify the monolayer, bilayer and trilayer graphene flakes [13.

The Non-contact mode AFM imaging (Figure 15. (B)) reveals that the graphene membrane adheres to the vertical wall of the hole for 2-10 $\mathrm{nm}$ due to van der Waals interaction with the substrate resulting in a considerable amount of pre-tension in the free-standing graphene membrane [13]. The 
elastic properties of graphene film were estimated by indenting the graphene membranes using AFM tips, as illustrated in Figure 15. (c). For indentation experiment two cantilevers with diamond tips of radii $27.5 \mathrm{~nm}$ and $16.5 \mathrm{~nm}$ were used [13. The force-displacement data for monolayer and
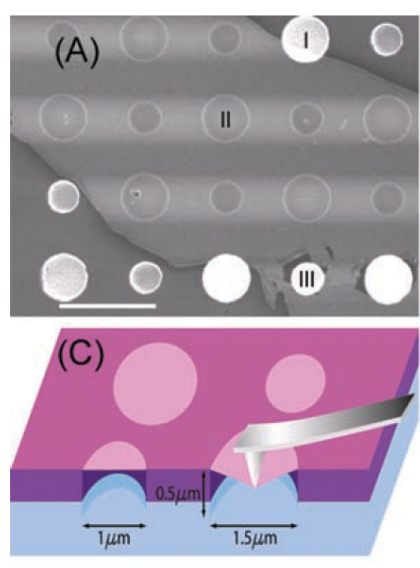
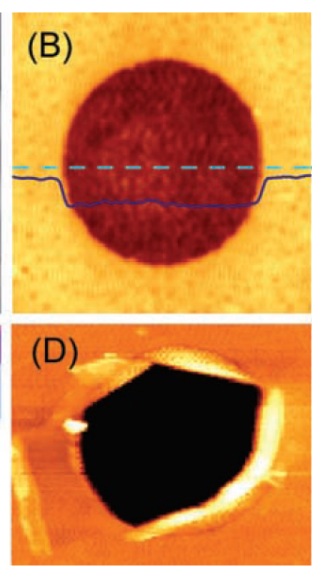

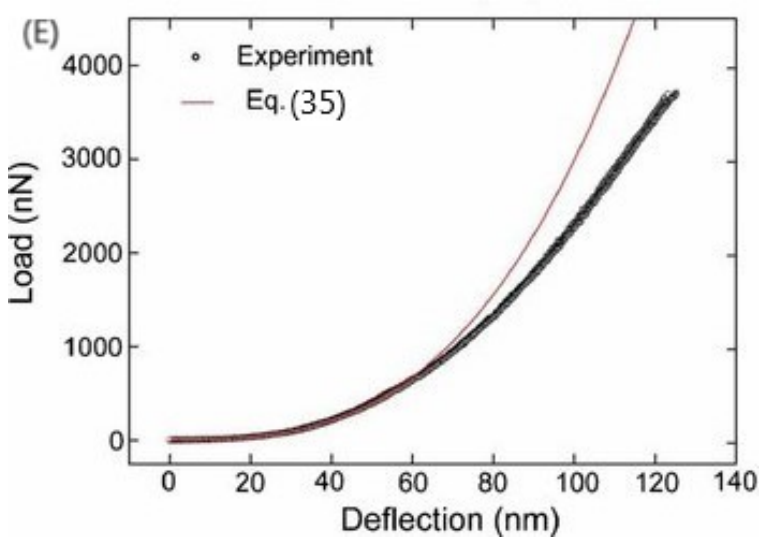

Figure 15: Experimental Results for the elastic properties of multilayer graphene [16]. (A) Images of suspended graphene membranes. (B) AFM image of monolayer graphene, $1.5 \mathrm{~mm}$ in diameter. (C) Image of AFM experimental setup. (D) AFM image of a fractured membrane [13. (E) Loaddisplacement data and curve fitting of Eqn. (35) of suspended bilayer graphene membranes [13.

bilayer graphene sample was then recorded to determine elastic stiffness and breaking stress [13. Lee et al. reported that, elastic modulus obtained for different flakes, tip radii, well depth, well radii and indentation speed and depth were statistically indistinguishable [13]. The force displacement curve for bilayer graphene follows equation (35) up to $50 \mathrm{~nm}$ deflection as seen in Figure 15 (E), 13. However curve shows a considerable amount of softening for displacement larger than $50 \mathrm{~nm}$ deflection and the experimental data falls significantly below the curve [13]. According to Lee et al., possibility of this phenomenon could be slip between graphene layers. The measured Young's modulus for 1, 2 and 3 layers of graphene were $340 \mathrm{Nm}^{-1}, 698 \mathrm{Nm}^{-1}$ and $986 \mathrm{Nm}^{-1}$ respectively [13. 


\section{Results and Discussion}

By perform nanoindentation experiment on multilayer graphene sheets, Lee et al. reported that strength of graphene reduces as we increase the number of layers from one to three [12]. By correlating the atomistic-continuum simulation with nanoindentation experiment conducted by Aleksey Falin et al. 9 and Lee et al. 13, we evaluated the elastic performance of multilayer graphene and revealed its deformation mechanism. We followed the load-displacement results reported by Lee et al [13. (Figure $15(\mathrm{E})$ ) and Aleksey Falin et al. 9] (Figure 14 (B)) for multi-layer graphene sheets using nanoindentation experiment. As our atomistic continuum model is limited to multi-layer graphene, (monolayer layer graphene can not be simulated), therefore, we compared the elastic properties of bilayer and tri-layer graphene with experimental results reported by Aleksey Falin et al [9] and Lee et al [13].

Because of large ratio of film radius to tip radius in experiment, clamped circular freeStanding membrane made up of linear isotropic elastic material under central point load is considered [13. For such a model, the relationship between indentation load and membrane deflection can be analytically expressed as [12]

$$
P=\sigma_{0}{ }^{2 D}(\pi a)\left(\frac{\delta}{a}\right)+E\left(q^{3} a\right)\left(\frac{\delta}{a}\right)^{3}
$$

where, $\mathrm{E}=$ Elastic Modulus, $\mathrm{t}=$ thickness, $\mathrm{a}=$ sample radius, $\mathrm{q}=$ function of Poisson's ratio $\nu, \mathrm{q}=$ $1 /\left(1.0491-0.1462 \nu-0.15827 \nu^{2}\right), \nu=$ Poisson's ratio $=0.17$. The pretension $\sigma_{0}$ and elastic modulus $\mathrm{E}$ of material are obtained by fitting the experimental results at non-linear elastic response at large deflection [12. The constitutive behavior of graphene layer undergoing small deformation can be assumed to have isotropic and linear elastic with young's modulus E and poisons ratio $\nu$ [12. The behavior of graphene under moderate to large deformation is often assumed to be nonlinear elastic (and model as continuum thin shell), the elastic response is expressed as [12]:

$$
\sigma=E \epsilon+D \epsilon^{2}
$$

Where E is Young's modulus and D is third-order elastic constant [16]. The Equation (35) is derived from the equation of strain energy given by $U=\frac{1}{2} \sigma \epsilon=E \epsilon^{2}+D \epsilon^{3}$, Where $\sigma=$ second order Piolo Kirchhoff stress, $\epsilon=$ Uni-axial Lagrange Strain.

\subsection{Atomistic Continuum Simulation Results to Reproduce Nano- indentation Experiment Conducted by Aleksey Falin et al.}

\subsubsection{Atomistic continuum simulations for suspended bi-layer graphene}

To reproduce the nanoindentation experiment conducted by Aleksey Falin et al. using atomistic continuum simulation, we carried out nanoindentation on bilayer and tri-layer graphene membrane freely suspended on soft elastic substrate. The graphene sheets are modelled as axisymmetric membrane of size $1310 \times 1310 \mathrm{~nm}^{2}$. A total of 1311 x 1311 axisymmetric membrane elements were used for meshing each graphene layer. In the simulation, square sheets are suspended on a circular hole of diameter $650 \mathrm{~nm}$ and probed at the center. Boundary conditions in the experiments conducted by Aleksey Falin et al. 9] and Lee et al. [13] are unknown, therefore we obtained simulation results for all the three boundary conditions mentioned in the section (4). We got a good match with the experiment using Boundary condition-1, possibly due to use of Equation (35) (The relationship between the indentation load and displacement of clamped circular thin plate) in this boundary condition.

Comparison of force-displacement curve obtained by atomistic continuum simulation with experiment for bilayer graphene is show in the Figure 14. Aleksey Falin et al., reported presence of a finite amount of pretension in suspended graphene sample tested experimentally [9]. However, 


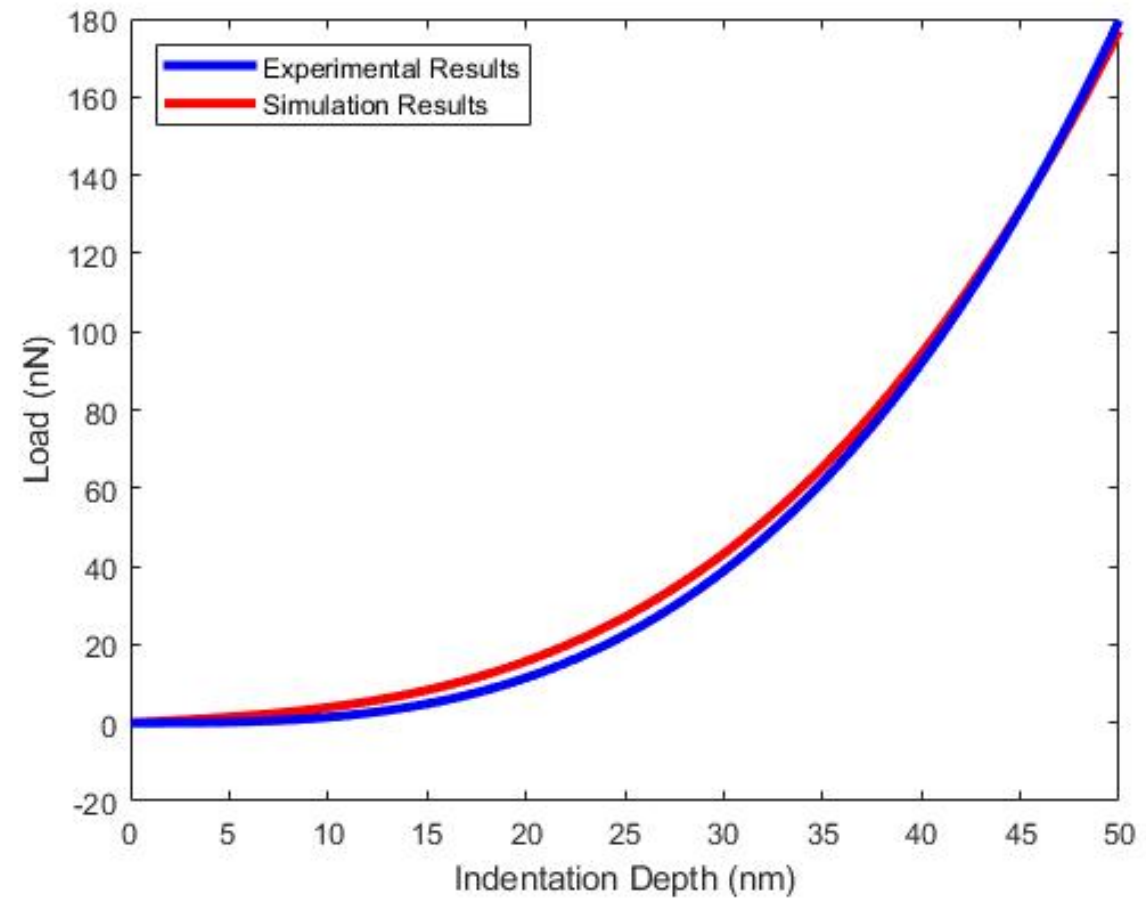

Figure 16: Comparison of Load - displacement curves for bi-layer graphene obtained from the simulation (Red curve) with the load-displacement curve reported in the experiment (blue curve) conducted by Aleksey Falin et al., 9] consider zero initial pretension.

in the atomistic continuum simulation, the graphene layer is under fully relaxed state and have zero initial pretension. Thus, we have shown the comparison of atomistic simulation results with experiment by eliminating pretension (i.e linear term in equation 35). The total time required to run this entire simulation using 8 nodes with 24 processors per node (Total 192 processors) was around 61 hours and 35 minutes (3695 minutes).

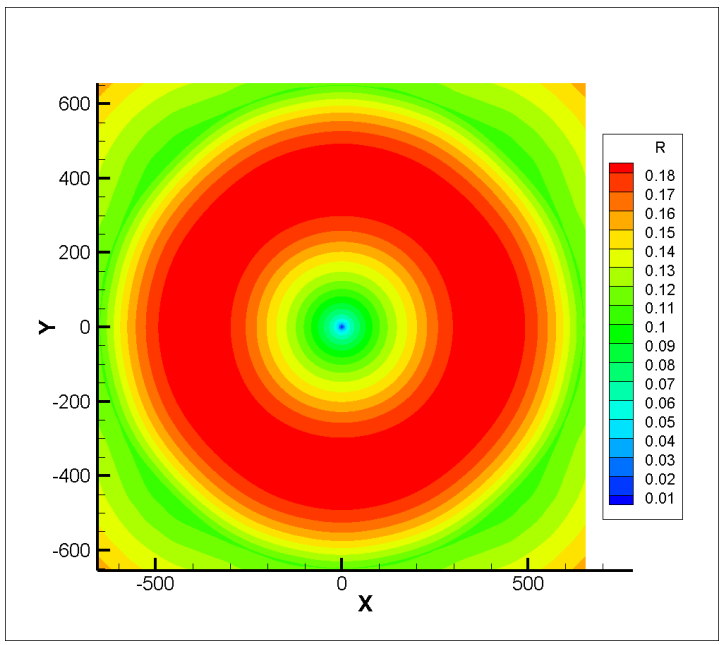

(a) 2D images of radial deformation

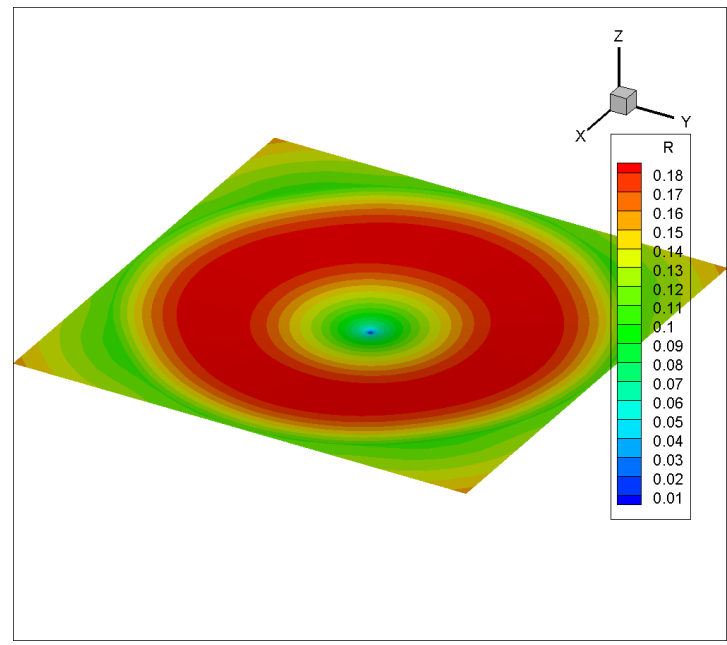

(b) 3D images of radial deformation 


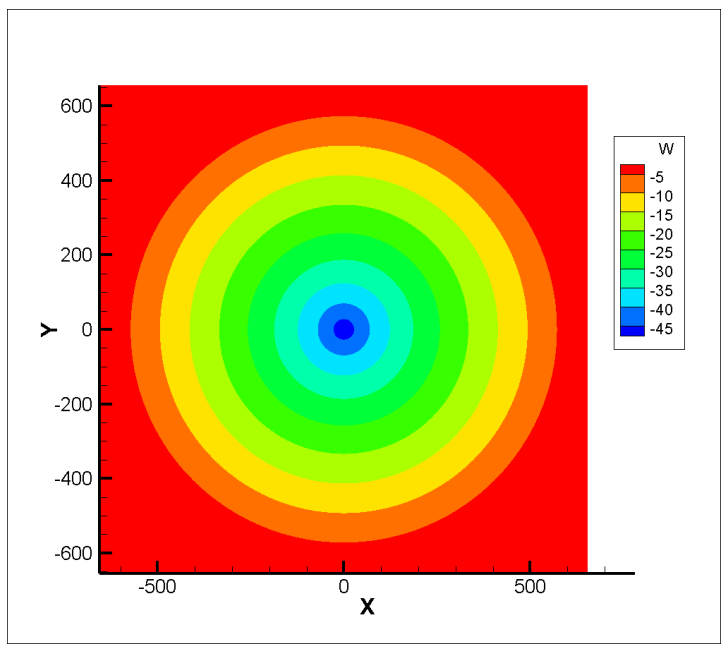

(c) 2D images of out-of-plane deformation

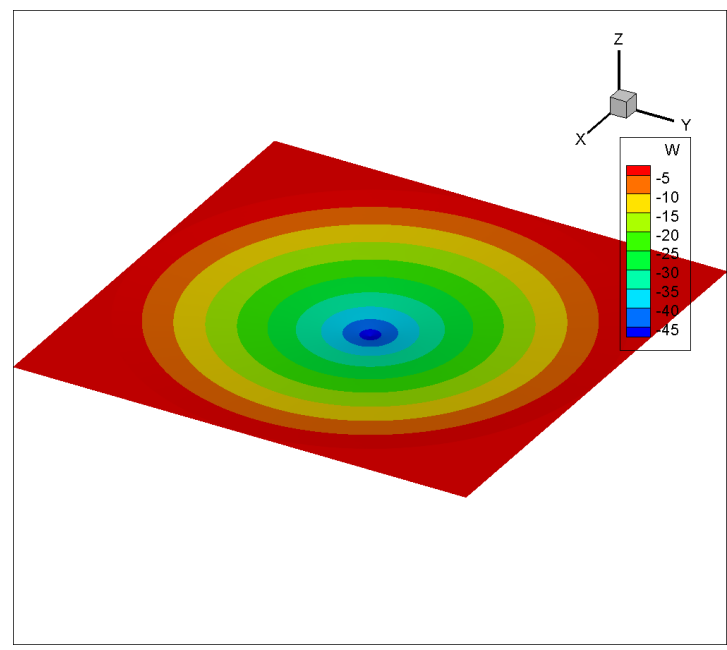

(d) 3D images of out-of-plane deformation

Figure 17: Post-processed results showing radial and out-of-plane deformation of bi-layer graphene for $50 \mathrm{~nm}$ indentation depth.

The force - displacement data obtained by AC simulation is superimposed on the experimental data (See Figure 16). This observation clearly shows that, the elastic modulus of bi-layer graphene is in good agreement with the experiment. The Young's modulus obtained by AC simulation is found to be approximately $644.5619 \mathrm{~N} / \mathbf{m}$ for bi-layer graphene, which is in a good agreement with Young's moduli reported in the experiment conducted by Aleksey Falin et al., $(645 \pm 16 \mathrm{~N} / \mathrm{m})$ 9]. The post processed results for out-of-plane deformation of bilayer graphene indented to $50 \mathrm{~nm}$ depth is shown in Figure 17.

\subsubsection{Atomistic continuum simulations for suspended tri-layer graphene}

Figure 18, shows comparison of load-displacement curve obtained by the atomistic continuum simulation with nanoindentation experiment for trilayer graphene. The graphene layer is under fully relaxed state and have zero initial pretension. The load - displacement curve obtained by the simulation is superimposed over the experimental results, clearly indicates that the elastic modulus of tri-layer graphene is in good agreement with the experiment. The Young's modulus obtained by AC simulation is found to be approximately $\mathbf{1 1 0 9 . 6} \mathbf{N} / \mathbf{m}$ for tri-layer graphene, which is in a good agreement with Young's moduli reported in the experiment conducted by Aleksey Falin et al (985 $\pm 10 \mathrm{~N} / \mathrm{m})$ [9].

The post processed results for out-of-plane deformation of trilayer graphene indented to 50 $\mathrm{nm}$ depth is shown in Figure 19. The deviation of force-displacement curves and difference between Young's modulus obtained from AC simulation and the experiment is possibly due to unknown boundary conditions and unknown Pre-tension in the actual experiment. The total time required to run this entire simulation using 8 nodes with 24 processors per node (Total 192 processors) was around 69 hours and 55 minutes (4195 minutes). 


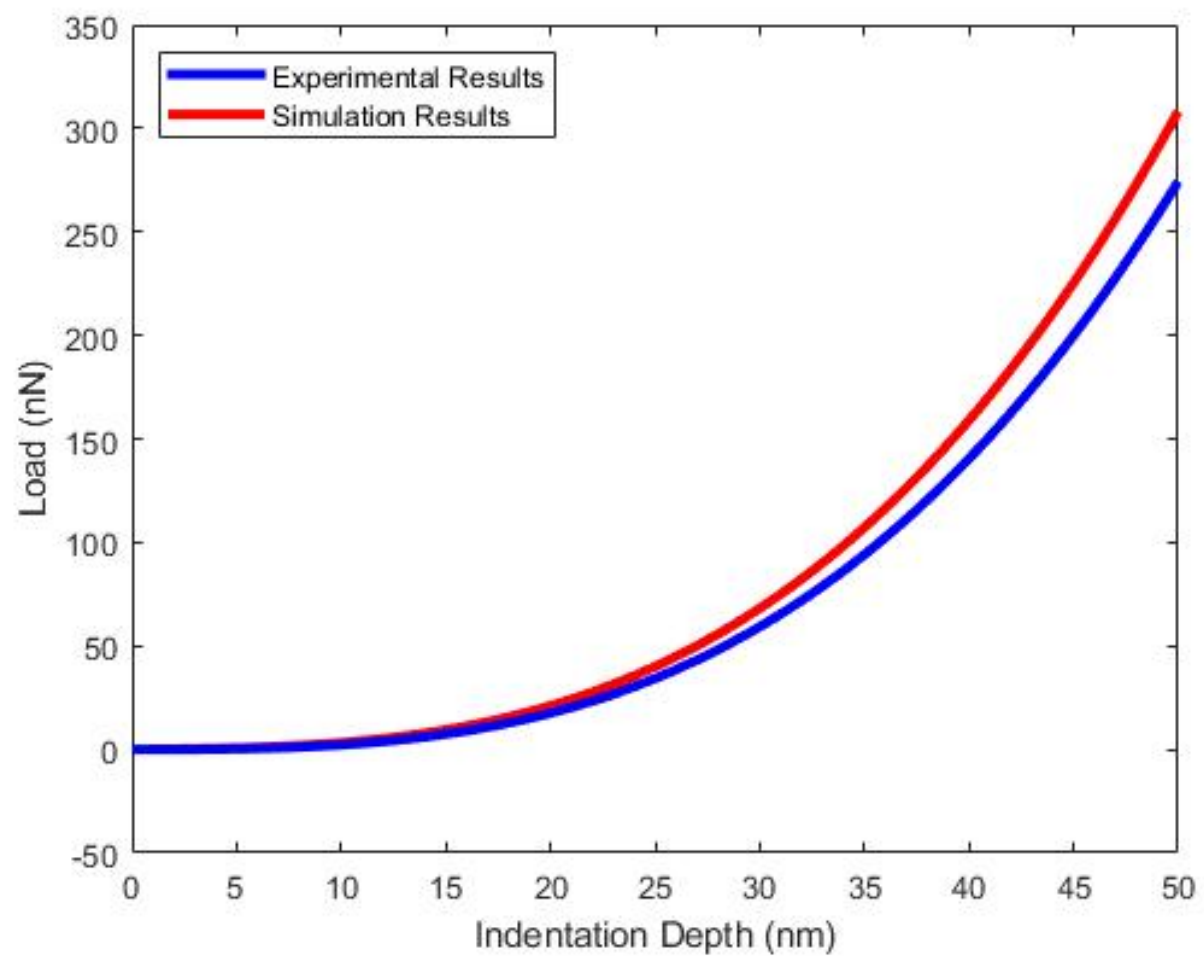

Figure 18: Comparison of Load - displacement curve for tri-layer graphene obtained from the simulation (Red curve) with the load-displacement curve reported in the experiment (blue curve) conducted by Aleksey Falin et al., [9] consider zero initial pretension.

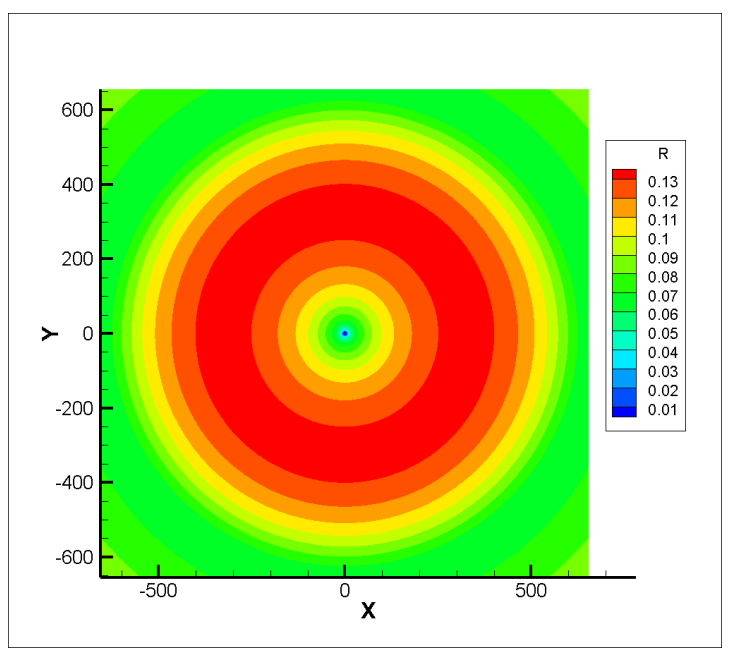

(a) 2D images of radial deformation

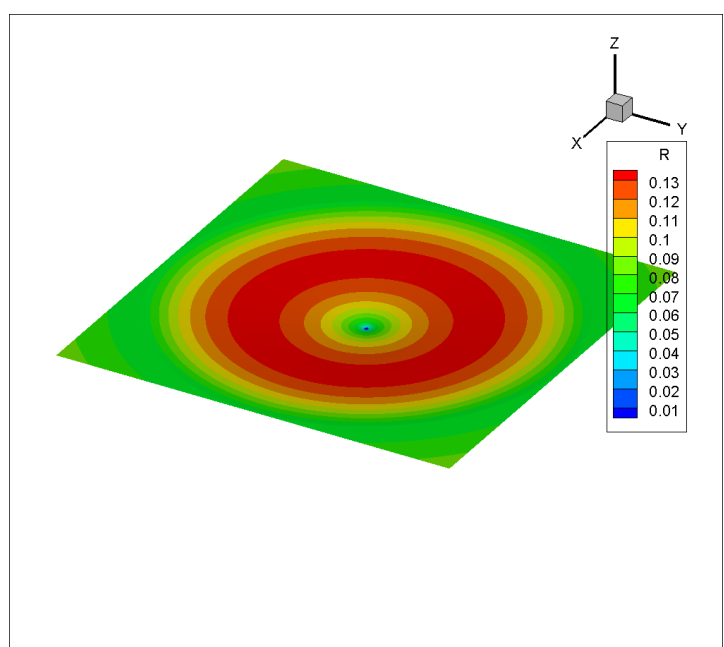

(b) 3D images of radial deformation 


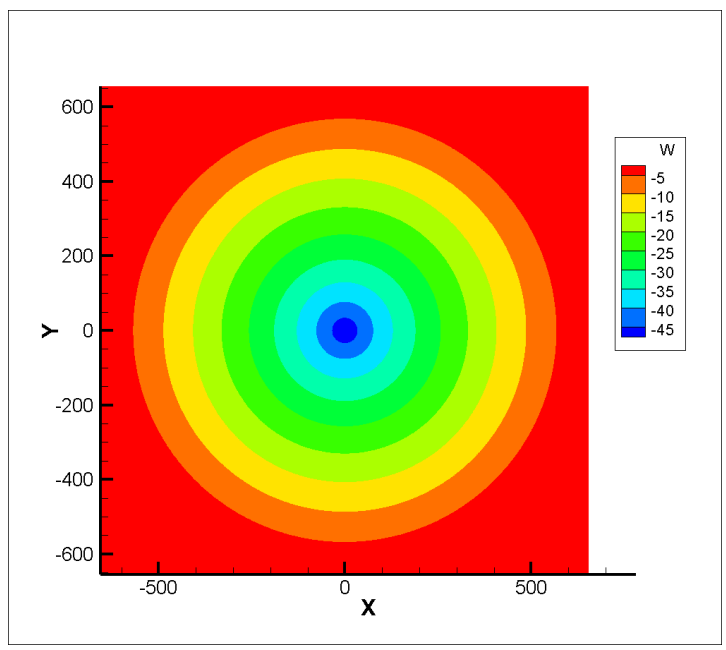

(c) 2D images of out-of-plane deformation

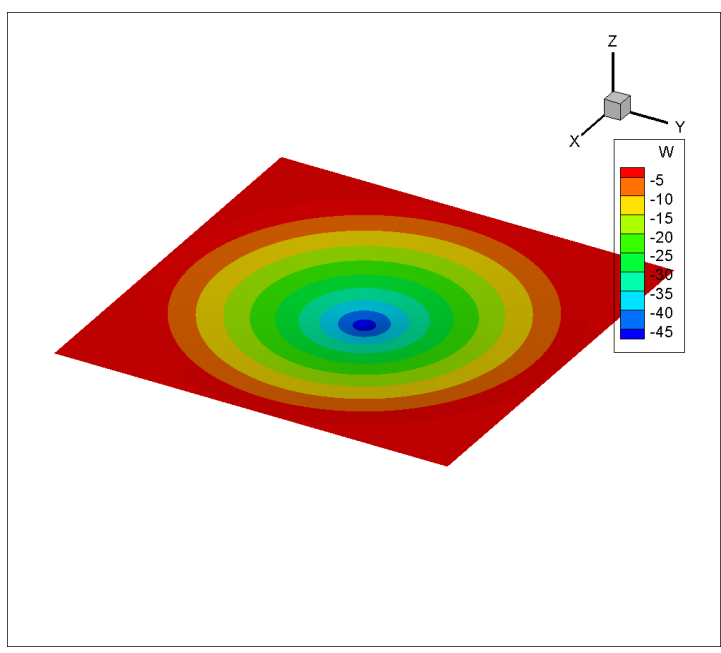

(d) 3D images of out-of-plane deformation

Figure 19: Post-processed results showing radial and out-of-plane deformation of tri-layer graphene for $50 \mathrm{~nm}$ indentation depth.

\subsection{Atomistic Continuum Simulation Results to Reproduce Nano- indentation Experiment Conducted by Lee et al.}

To compare the nanoindentation experiment conducted by Lee et al. (2009), using atomistic continuum simulation, we carried out nanoindentation on bilayer graphene sample freely suspended on soft elastic substrate. The graphene sample is modelled as axisymmetric membrane of size 1510 X $1510 \mathrm{~nm}^{2}$. A total of 1001 X 1001 axisymmetric membrane elements were used for meshing. In the simulation, square sheets are suspended on a circular hole of diameter $750 \mathrm{~nm}$ and probed at its center. The area outside the suspended region is fixed (Boundary Condition-1 given in section 4). Comparison of load-displacement curve obtained by atomistic continuum simulation with experiment for bilayer graphene is show in the Figure 15 (E). Lee et al., reported presence of a finite amount of pretension in suspended graphene sample tested experimentally [13]. However, in atomistic continuum simulation, the graphene layer is under fully relaxed state and have zero initial pretension. Thus, we have shown the comparison of atomistic simulation results with experiment by eliminating pretension (i.e linear term in equation 35).

Force - displacement data obtained by the simulation is superimposed over the experimental results (see Figure 20), indicating the elastic modulus of tri-layer graphene is in good agreement with the experiment. The Young's modulus obtained by AC simulation is found to be approximately 790.4883 N/m, which is in a good agreement with Young's moduli reported in the experiment conducted by Lee et al. [13] for bilayer graphene sample. The post processed results for out-of-plane deformation of bilayer graphene indented to $120 \mathrm{~nm}$ depth is shown in Figure 21. The deviation of force-displacement curves and difference between Young's modulus obtained from AC simulation and the experiment is possibly due to unknown boundary conditions and unknown pre-tension in the actual experiment. The total time required to run this entire simulation using 8 nodes with 24 processors per node (Total 192 processors) was around 110 hours and 25 minutes (6625 minutes). 


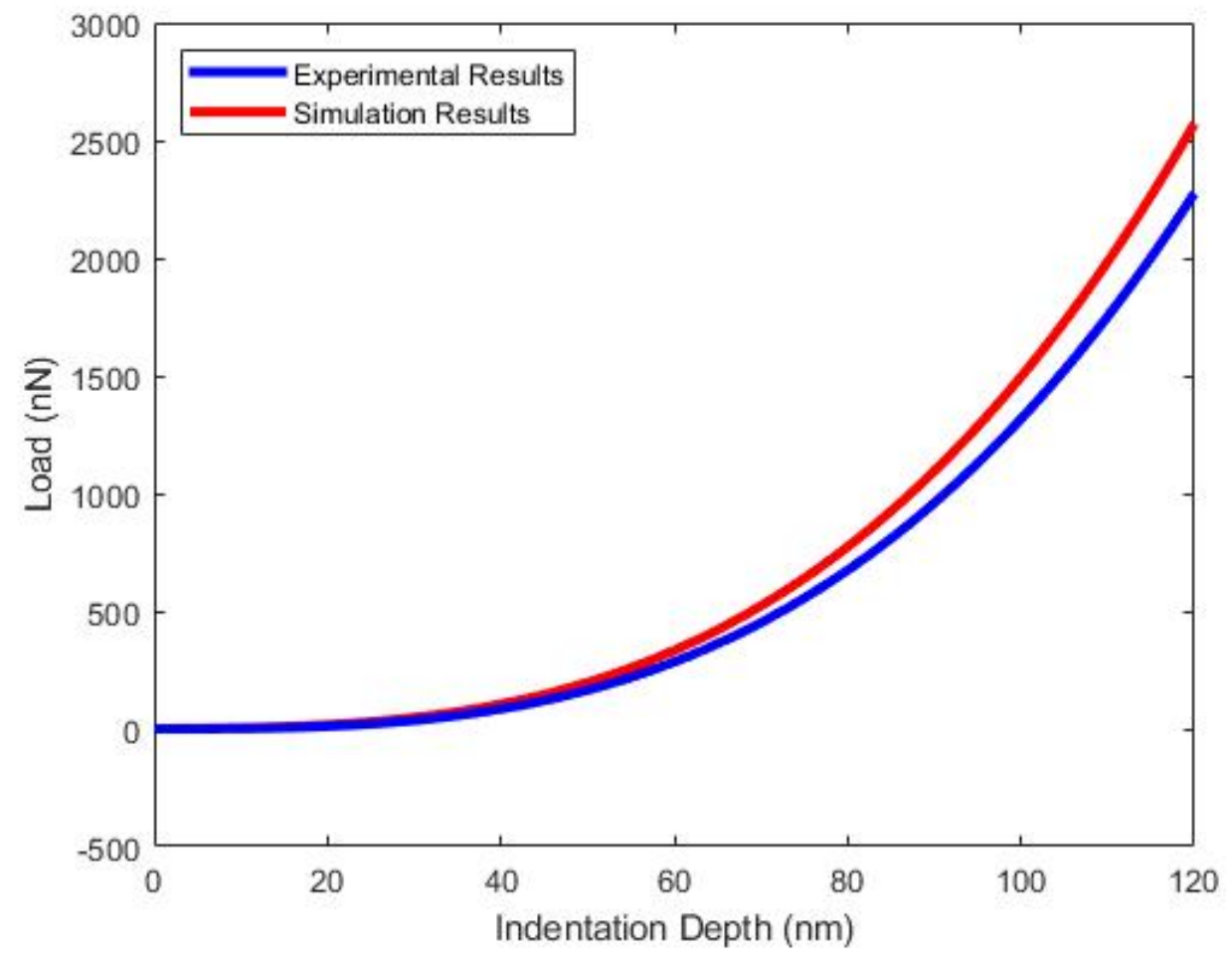

Figure 20: Comparison of Load - displacement curve for bi-layer graphene obtained from the simulation (Red curve) with the load-displacement curve reported in the experiment (blue curve) conducted by Lee et al., [13] consider zero initial pretension.

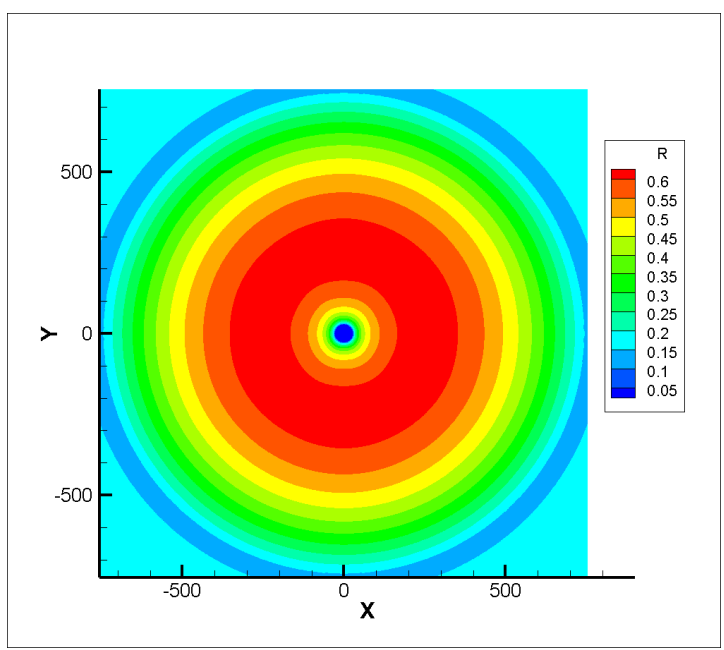

(a) 2D images of radial deformation

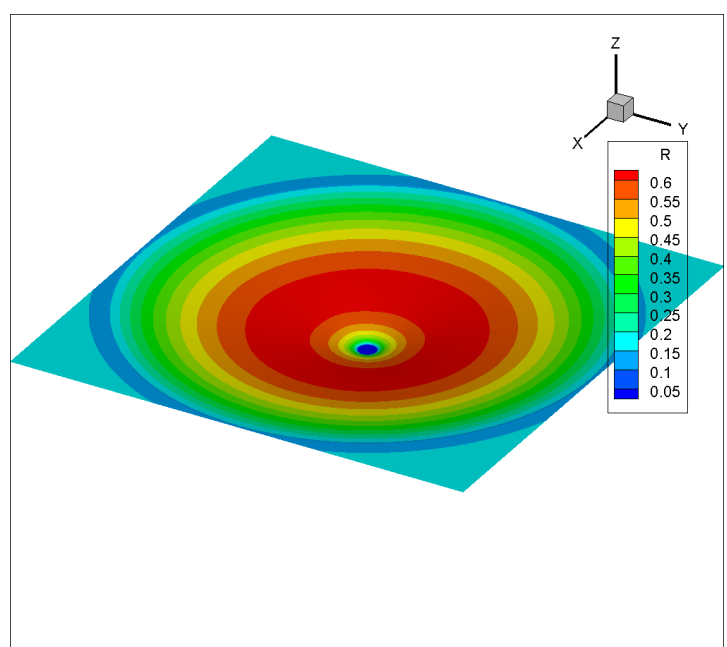

(b) 3D images of radial deformation 


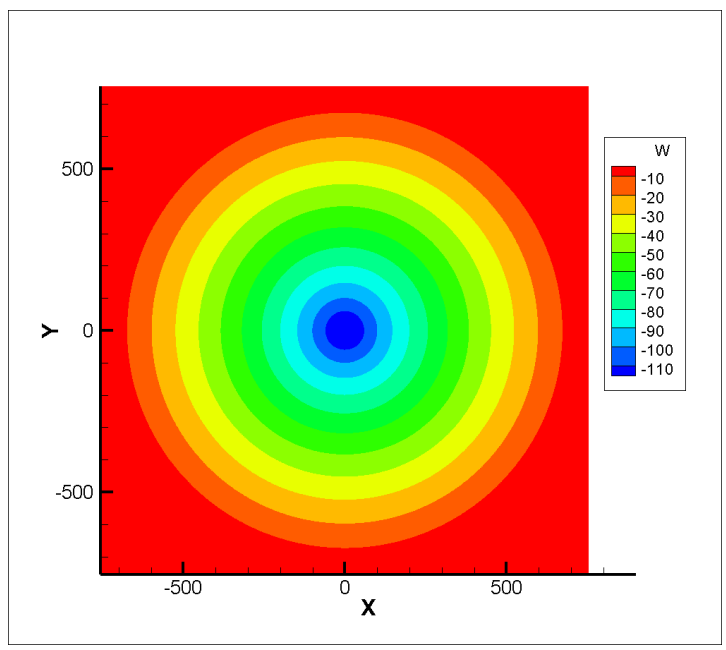

(c) 2D images of out-of-plane deformation

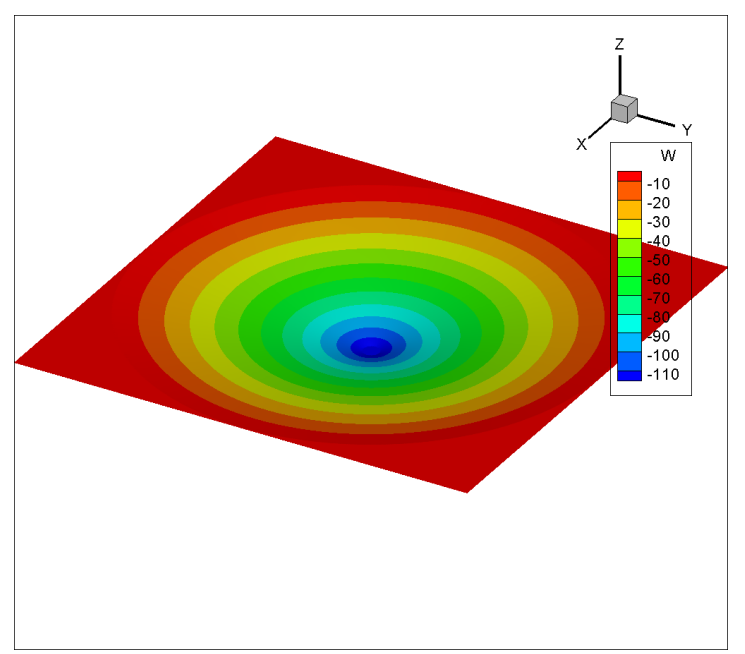

(d) 3D images of out-of-plane deformation

Figure 21: Post-processed results showing radial and out-of-plane deformation of bi-layer graphene at $120 \mathrm{~nm}$ indentation depth.

\subsection{Computational Platform}

\subsubsection{Nanoindentation Simulation}

All simulations involving nanoindentation were run using Comet, a dedicated XSEDE cluster with 24 Cores/node operated by the San Diego Supercomputer Center at UC San Diego and available through Extreme Science and Discovery Environment (XSEDE) program. Each compute node of comet HPC system has an Intel 24-Cores Xeon E5-2680v3 processor (24:75 MB cache, running at 2:5 $\mathrm{GHz}$ ), $128 \mathrm{~GB}$ of DRAM and a $400 \mathrm{~GB}$ of Solid-State Drive (SSD). Compilation of Fortran routines was carried out using intel application of interface version 2018.1.163.

\subsubsection{Uniaxial and Biaxial Compression Simulation}

All simulations involving uniaxial and biaxial compression were run using Michigan Tech's shared high-performance computing infrastructure, Superior. Its Generation 2.0 consist of 85 CPU compute nodes, each having Intel $32 \mathrm{CPU}$ cores Xeon E5-2683, running at $2.10 \mathrm{GHz}, 256$ GB RAM and providing 91 TFLOPS. Compilation of Fortran routines was carried out using Intel custom parallel program (MPICH + Intel 2016.1). The total time required to run each simulation using $96 \mathrm{CPU}$ processors was 3 hours, 22 minutes.

\subsubsection{Post Processing}

MATLAB version 2020a was used for reading the data files, curve fitting and plotting graphs for the simulations. Tecplot 360, version 2019 R1 was used to produce the post-processed images of deformed shapes of graphene. The underlying numerical data for load-displacment curve reported in the experiments was extracted for comparison purpose using WebPlotDigitizer software version 4.4 [21]. 


\section{Uniaxial and Biaxial Compression of Graphene to Study the Surface Morphology:}

Crumpled graphene films possess many novel properties due which they are extensively used in the applications like biomechanics, electronics, energy storage and composites. [26]. Zang et al. reported a method to control crumpling of graphene that can be used as conductive coating on electrodes to accomplish superhydrophobic property. The amount of crumpling affects the performance of graphene in graphene-based devices and materials [26. Thus, controlling these surface morphologies is extremely important to control the performance of graphene in the graphene-based devices [26]. Ripples and wrinkles formation on supported graphene has been reported by (Zhang, K. and M. Arroyo (2013)) [25].

To study the surface morphology of graphene sheet, here we report the deformed shapes of the bilayer graphene under uniaxial and biaxial compression 25. The surface morphology of graphene film under uniaxial compression is distinct from that of biaxial compression. We observe that graphene layers show wrinkles with smooth undulations and crumpling with sharp folds, ridges and vertices [25. Graphene layers shows widely spread wrinkles of larger amplitudes for weaker adhesion potential $\left(0.096 \mathrm{aJ} / \mathrm{nm}^{2}\right)$ between graphene layers, while densely spaced small amplitude wrinkles can be seen on graphene sample for stronger inter-layer adhesion potential $\left(0.96 \mathrm{aJ} / \mathrm{nm}^{2}\right)$.
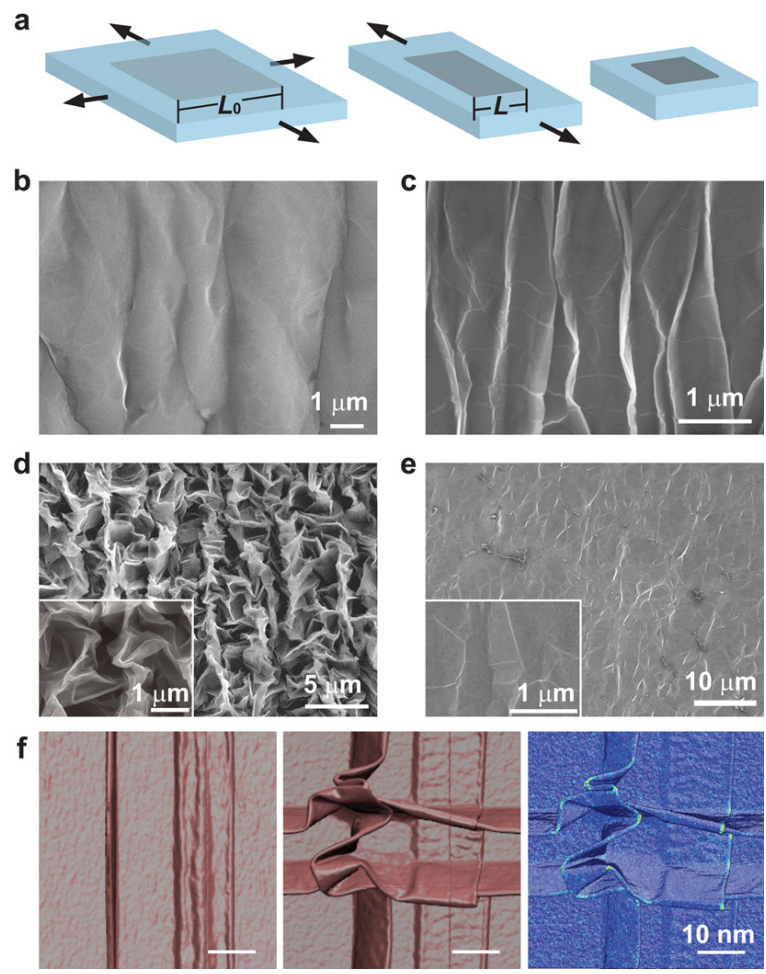

Figure 22: a, schematic illustration of deformation of a graphene membrane on a biaxially prestretched substrate followed by relaxation of the substrate. b-e, SEM images of different morphologies generated on the graphene sheet: (b) shows wrinkles formation, then formation of delaminated buckles formation as the substrate relaxed uniaxially (c), followed by the formation of crumples as the substrate is relaxed biaxially (d), followed by unfolding of graphene as the substrate is biaxially stretched back (e). (f) Atomistic simulation results of the crumpling of a single-layer graphene under uniaxial and biaxial compression. Nat. Mater. 12 (2013) 321-325 [25]. 


\subsection{Simulation Results}

Graphene of $500 \mathrm{~nm} \times 500 \mathrm{~nm}$ bilayer samples are considered for simulations purpose. A total of 501 x 501 non-linear axisymmetric membrane elements are used. Ripples formation on the graphene film is then studied under uniaxial and biaxial compression. Initially graphene seems to be planar until a threshold point after which it develops periodic, small amplitude ripples, see Figure (22). Under uniaxial compression, graphene develops a pattern of parallel delaminated buckles [26. On biaxial compression, these delaminated buckles are compressed along the ridges and from crumpled surface morphology [27, see Figure $(25,26)$. An intersecting crumpling pattern is formed due to the intersection of two orthogonal buckles [27. Amplitude and arrangement of wrinkles formed on the surface largely depends upon inter-layer adhesion potential. In case of high inter-layer adhesion potential $\left(0.96 \mathrm{aJ} / \mathrm{nm}^{2}\right)$, dense wrinkles of smaller amplitudes are observed. However, at low interlayer adhesion potential $\left(0.096 \mathrm{aJ} / \mathrm{nm}^{2}\right)$, widely spread wrinkles of larger amplitudes are formed on the graphene surface. The results of atomistic simulation reveal high stress concentration around the ridges and vertices [27. There are not much experimental results supporting the distributed ripples associated to compression of graphene layers [27.

\subsection{Observation and Discussion}

1. Our large-scale atomistic simulations results observe multiple morphological deformation phases in bilayer graphene under uniaxial and biaxial compression depending upon van der Waals interaction between graphene layers.

2. We study the variation of height and quantity of spontaneous localized wrinkles upon anisotropic uniaxial and biaxial compression w.r.t inter-layer adhesion potential, see Figure 27.

3. Smaller amplitude ripples are developed on graphene sheets due to strong inter-layer adhesion potential $\left(0.96 \mathrm{aJ} / \mathrm{nm}^{2}\right)$, see Figure 24, 26.

4. Ripple height increases with decrease in inter-layer adhesion potential $\left(0.096 \mathrm{aJ} / \mathrm{nm}^{2}\right)$, see Figure 23, 25.

5. As we increase the compression $(3 \%)$, the small amplitude ripples are formed which undergoes partial release of rippling in their vicinity [27].

6. At the junctions where these wrinkles meet under biaxial compression, an abundance of complex T-junction can be seen forming complex structures at these junctions [27, see Figure 25 .

7. We have chosen equilibrium spacing of $0.6 \mathrm{aJ} / \mathrm{nm}^{2}$ to better visualize the ripples and wrinkle formed, which results in compliant interaction.

8. These simulation results can be used to study and control the wrinkling network by compressing supported graphene to improve graphene performance in graphene-based devices. 


\subsection{Results of Uniaxial Compression:}

\subsubsection{Weak Adhesion Potential Between Graphene Layers (0.096 aJ/nm2).}

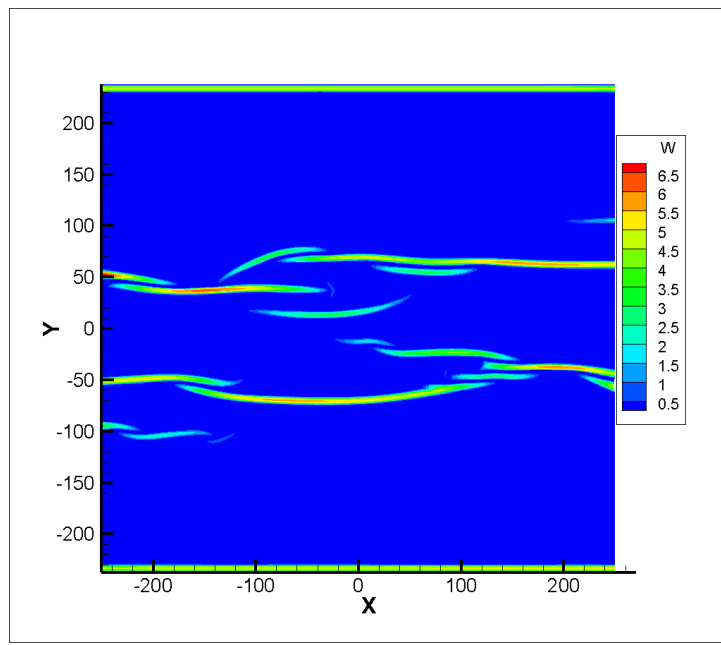

(a) 2D images of out-of-plane deformation

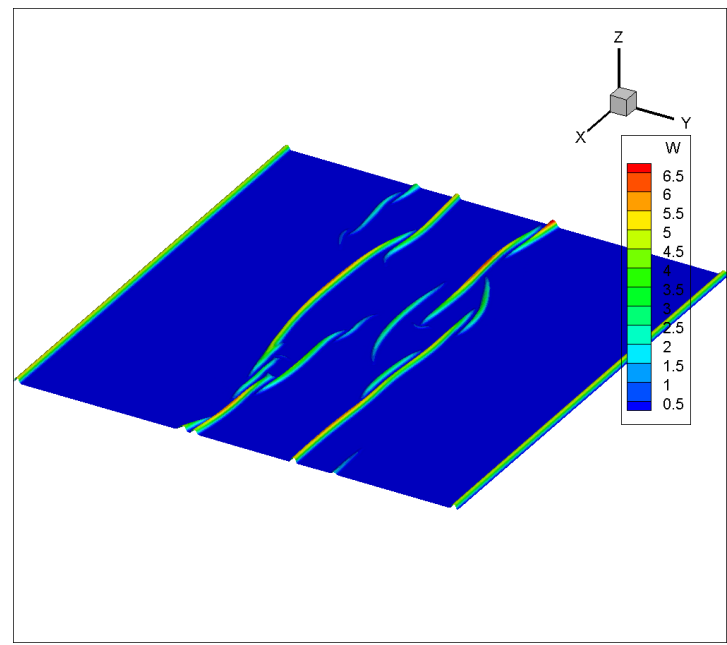

(b) 3D images of out-of-plane deformation

Figure 23: Post processed images of atomistic simulation results showing ripples and wrinkles formation on graphene layers under $5 \%$ uniaxial compression strain and weak adhesion potential.

\subsubsection{Strong Adhesion Potential Between Graphene Layers (0.96 aJ/nm2).}

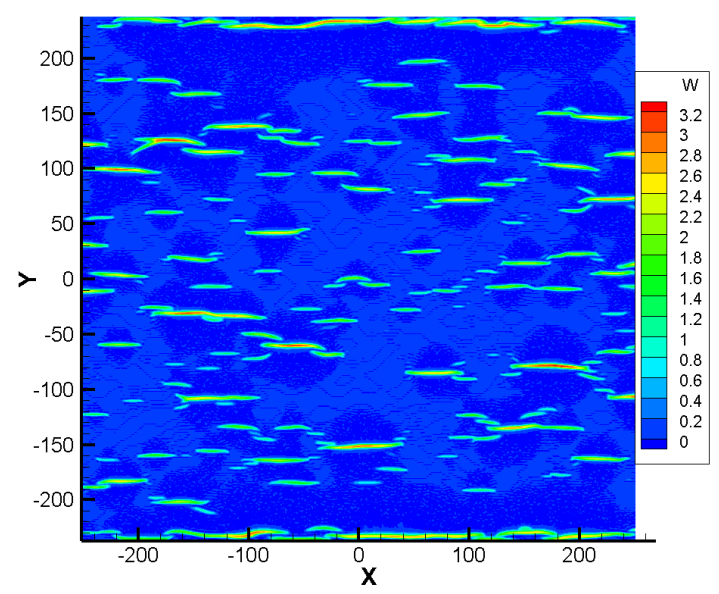

(a) 2D images of out-of-plane deformation

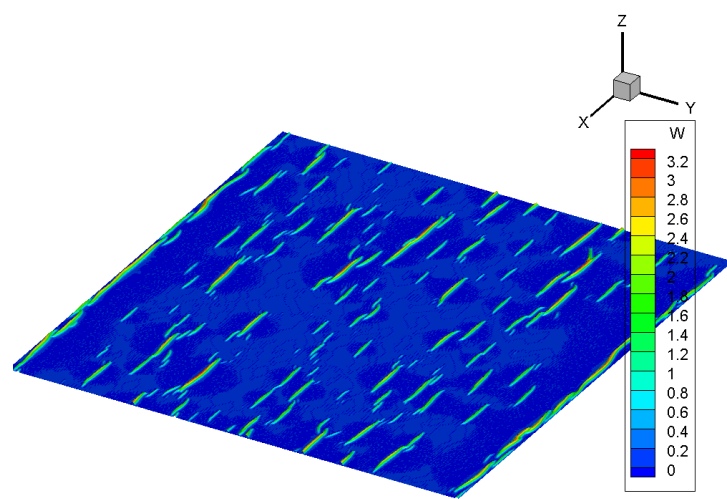

(b) 3D images of out-of-plane deformation

Figure 24: Postprocessed images of atomistic simulation results showing ripples and wrinkles formation on graphene layers under $5 \%$ uniaxial compression strain and strong adhesion. 


\subsection{Results of Biaxial Compression:}

7.4.1 Weak Adhesion Potential Between Graphene Layers (0.096 aJ/nm2).

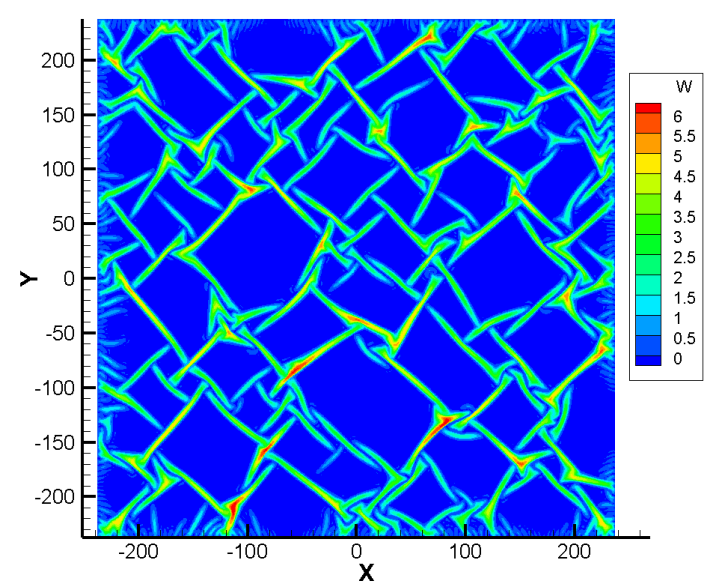

(a) 2D images of out-of-plane deformation

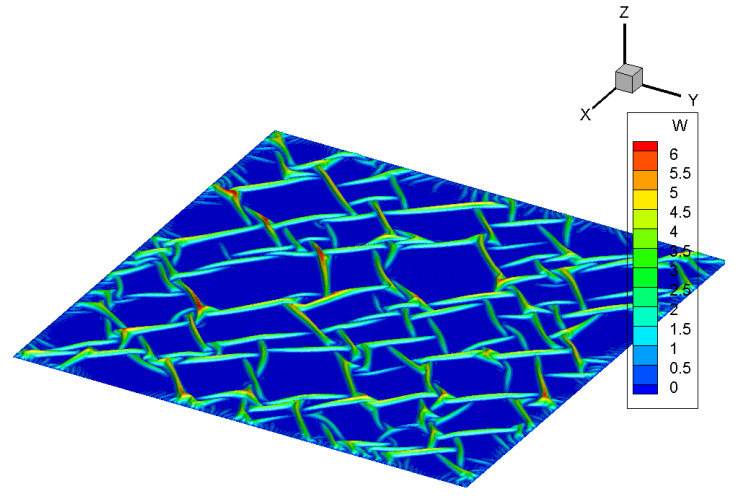

(b) 3D images of out-of-plane deformation

Figure 25: Postprocessed images of atomistic simulation results showing ripples and wrinkles formation on graphene layers under $5 \%$ biaxial compression strain and weak adhesion.

\subsubsection{Strong Adhesion Potential Between Graphene Layers (0.96 aJ/nm2).}

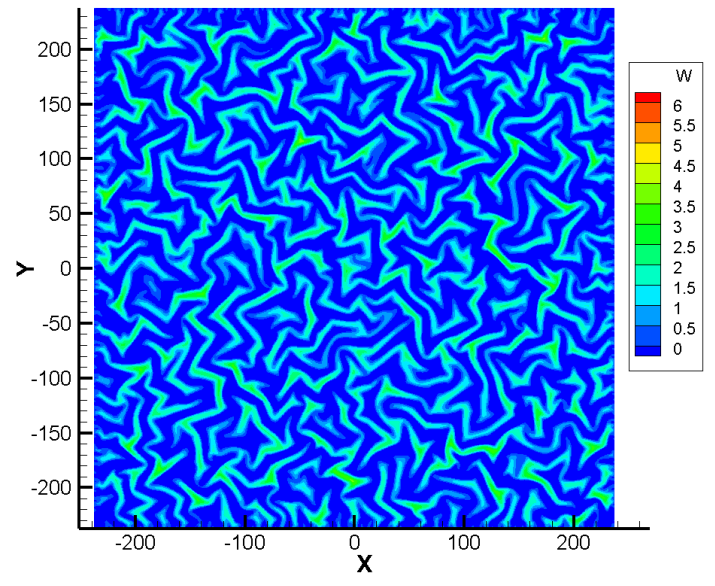

(a) 2D images of out-of-plane deformation

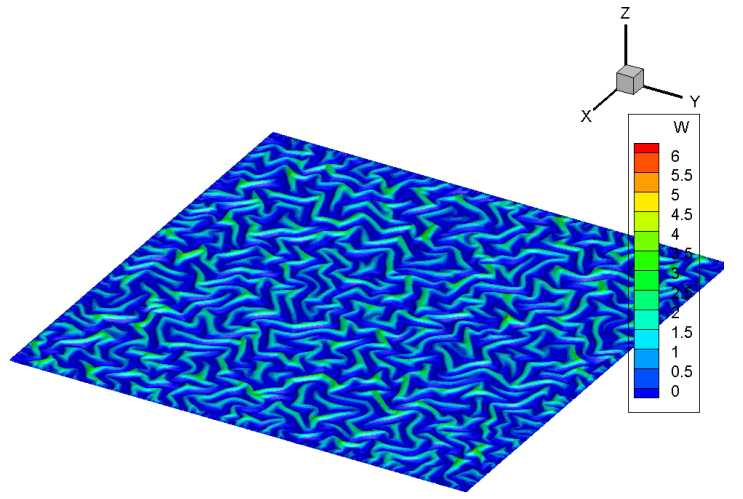

(b) 3D images of out-of-plane deformation

Figure 26: Postprocessed images of atomistic simulation results showing ripples and wrinkles formation on graphene layer under $5 \%$ biaxial compression strain and strong adhesion. 


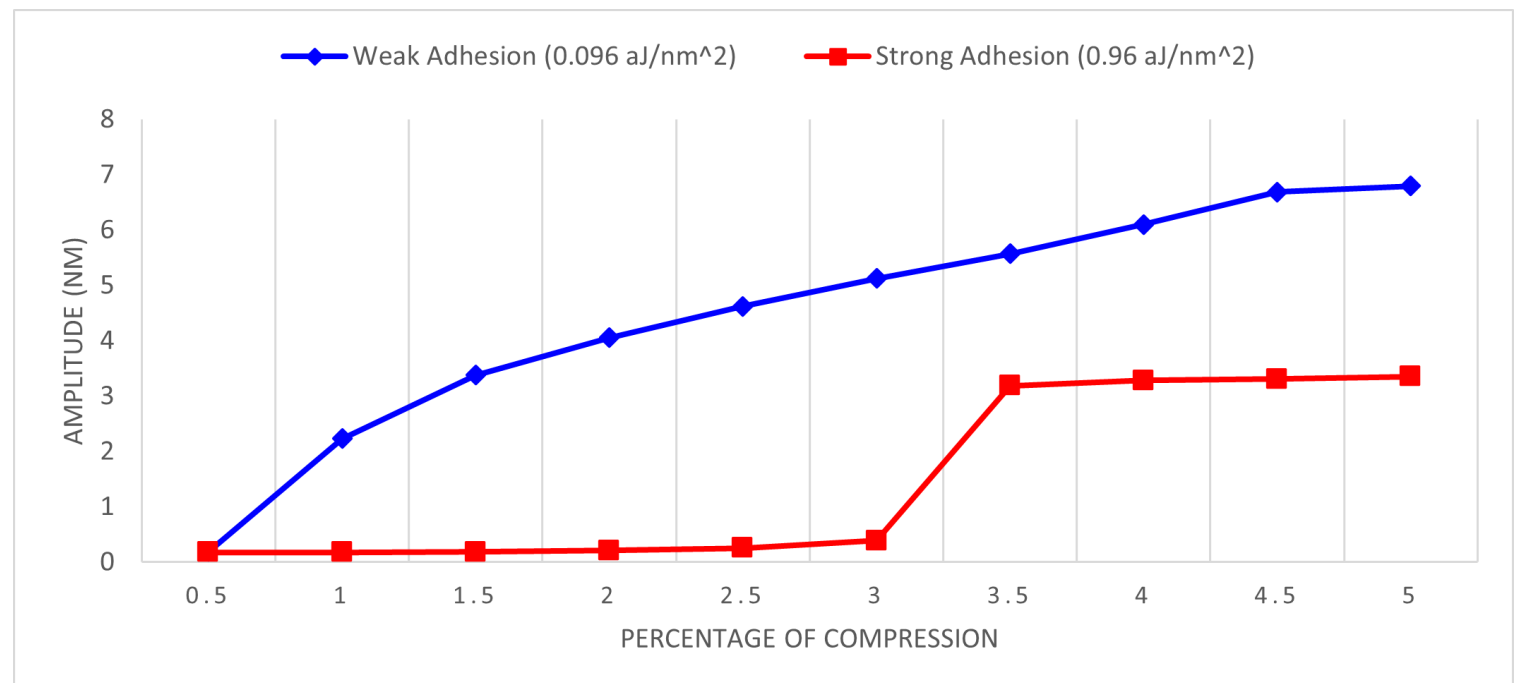

(a) Amplitude - uniaxial compression strain (0-5\%) plot for weak and strong inter-layer adhesion potential

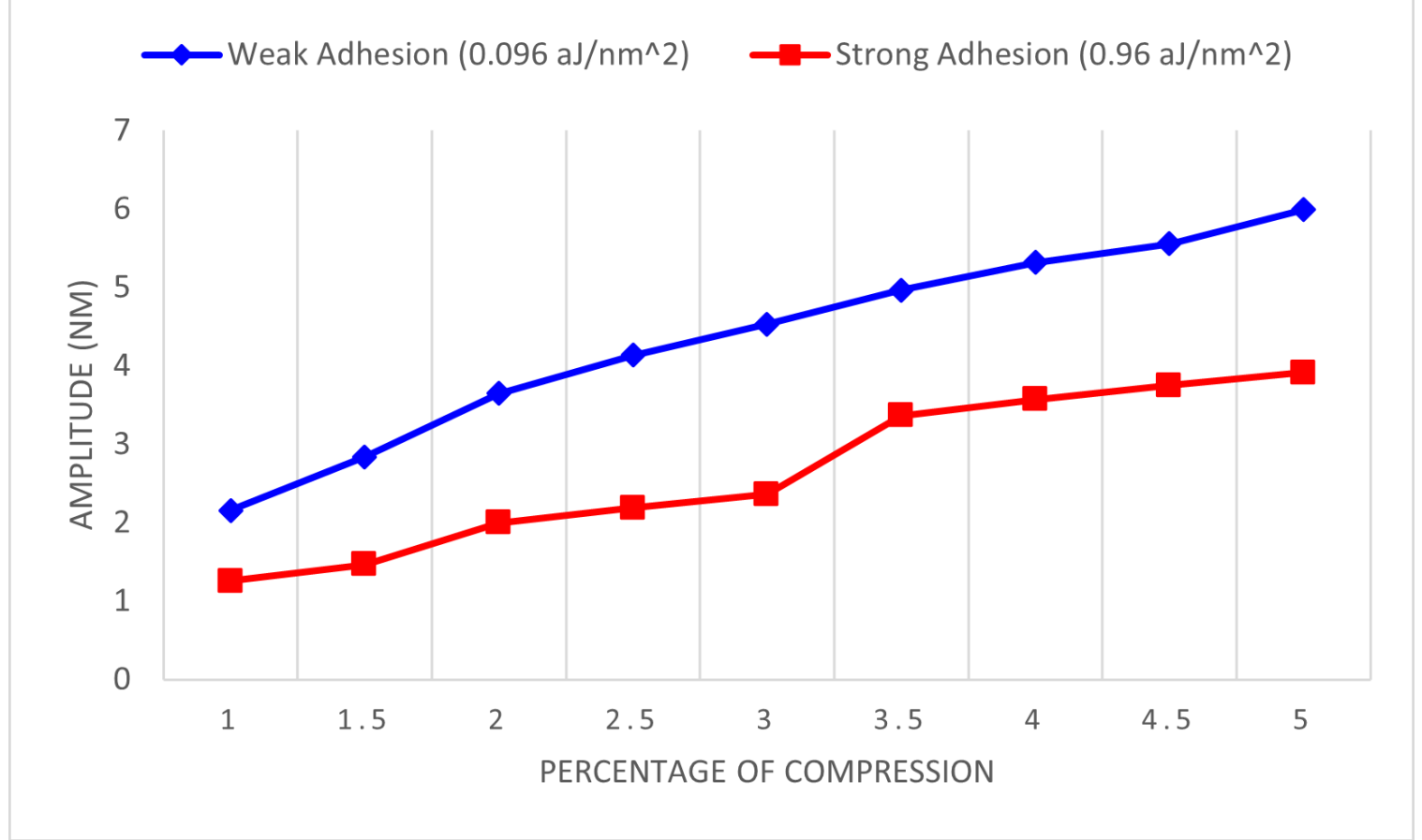

(b) Amplitude - biaxial compression strain (0-5\%) plot for weak and strong inter-layer adhesion potential

Figure 27: Ampltitude - compression plots for bilayer graphene subjected to uniaxial and biaxial compression strain at different adhesion potential 


\section{Future Scope}

\section{Nano-indentation}

1. Obtain optimal boundary condition to minimize the error in atomistic continuum simulation results in comparison with the experiments.

2. Obtain wrinkling pattern at each displacement of indentation depth.

3. Study the effect of indenter radius, pre-tension, number of graphene layers on the elastic and frictional properties of graphene.

\section{Uniaxial/Biaxial Compression}

1. Study the effect of substrate Van der Waals interaction with the graphene layers.

2. Study the effect of friction between substrate and graphene.

3. Study the effect of number of graphene layers on the elastic properties like intrinsic breaking strength, elastic modulus and frictional properties. 


\section{Conclusion}

1. Direct reproduction of the nano-indentation experiment through atomistic simulations has been a major challenge due to the large size of graphene samples tested experimentally.

2. The greatest drawback of MD, DFT, and Quantum mechanics simulation is its inconsistency in modeling monolayer and multilayer graphene samples greater than $30 \mathrm{~nm}$ in radius making them computationally expensive and prohibitive to simulate large-scale graphene samples [23].

3. This atomistic continuum model developed by Ghosh and Arroyo [10, can simulate large-scale graphene accurately, making it best suitable for the simulation the graphene samples which are often used in experiments and real-world applications.

4. This work reports the comparison of atomistic continuum simulation results for nanoindentation with the experiments conducted by Lee et al., and Aleksey Falin et al. Simulation results of bilayer and trilayer graphene up to $50 \mathrm{~nm}$ indentation depth yields in-plane Young's modulus of $644.5619 \mathrm{~N} / \mathrm{m}$ and $1109.6 \mathrm{~N} / \mathrm{m}$ respectively (Bulk Young's modulus $=0.9078 \mathrm{TPa}$ and $1.041 \mathrm{TPa}$ respectively which falls within a range of the experimental results reported by Aleksey Falin et al., 9 .

5. Simulations on bilayer graphene for $120 \mathrm{~nm}$ indentation depth yields in-plane Young's modulus of $790.4883 \mathrm{~N} / \mathrm{m}$ (Bulk Young's modulus = $1.11 \mathrm{TPa}$ ) which falls within a range of the experiment reported by Lee et al., [13].

\begin{tabular}{|c|c|c|c|}
\hline Graphene Samples & Experiment (N/m) & Simulation (N/m) & Error (\%) \\
\hline Bilayer (Falin et al.) & $645 \pm 16$ & 644.5619 & 0 \\
\hline Trilayer (Falin et al.) & $985 \pm 10$ & 1109.6 & 10.32 \\
\hline Bilayer (Lee et al.) & 698 & 790.4883 & 11.7 \\
\hline
\end{tabular}

6. The deviation of force-displacement curves and difference between Young's modulus obtained from AC simulation and the experiment is possibly due to unknown boundary conditions and unknown pre-tension in the actual experiment.

7. Pretension value highly manipulates the load-displacement plot. We note that while there is a finite amount of pretension in the experiments, in simulations the graphene samples are fully relaxed and have no initial tension.

8. The energy at 12000 iterations is better converged than 1000 iterations. Therefore, we ran the simulations of nano-indentation for 12000 iterations (for each load-step) to get fully converged and accurate results.

9. Our large-scale atomistic simulations observe spontaneous localized wrinkles in bilayer graphene under uniaxial and biaxial compression depending upon adhesion (van der Waals) interactions between graphene layers.

10. Smaller amplitude ripples are developed in graphene samples for high inter-layer adhesion potential. Besides, ripple amplitudes increase with the decrease in inter-layer adhesion potential. 


\section{References}

[1] Deji Akinwande, Christopher J. Brennan, J. Scott Bunch, Philip Egberts, Jonathan R. Felts, Huajian Gao, Rui Huang, Joon Seok Kim, Teng Li, Yao Li, Kenneth M. Liechti, Nanshu Lu, Harold S. Park, Evan J. Reed, Peng Wang, Boris I. Yakobson, Teng Zhang, Yong Wei Zhang, Yao Zhou, and Yong Zhu. A review on mechanics and mechanical properties of 2D materials - Graphene and beyond. Extreme Mechanics Letters, 13:42-77, 2017.

[2] M. Arroyo and T. Belytschko. Finite element methods for the non-linear mechanics of crystalline sheets and nanotubes. International Journal for Numerical Methods in Engineering, 59(3):419$456,2004$.

[3] Richard H. Byrd, Peihuang Lu, Jorge Nocedal, and Ciyou Zhu. A Limited Memory Algorithm for Bound Constrained Optimization. SIAM Journal on Scientific Computing, 16(5):1190-1208, 1995 .

[4] Guoxin Cao and Huajian Gao. Mechanical properties characterization of two-dimensional materials via nanoindentation experiments. Progress in Materials Science, 103(June 2018):558-595, 2019.

[5] Andres Castellanos-Gomez, Menno Poot, Gary A. Steele, Herre S.J. Van Der Zant, Nicolás Agraï, and Gabino Rubio-Bollinger. Elastic properties of freely suspended MoS2 nanosheets. Advanced Materials, 24(6):772-775, 2012.

[6] Wei-ta Chu. Chapter 9 Newton's Method. pages 1-27, 2014.

[7] Antonio Coppola and Brandon M Stewart. lbfgs : Efficient L-BFGS and OWL-QN Optimization in $\mathrm{R}$.

[8] Zhaohe Dai, Nanshu Lu, Kenneth M. Liechti, and Rui Huang. Mechanics at the interfaces of 2D materials: Challenges and opportunities. Current Opinion in Solid State and Materials Science, 24(4), 2020.

[9] Aleksey Falin, Qiran Cai, Elton J.G. Santos, Declan Scullion, Dong Qian, Rui Zhang, Zhi Yang, Shaoming Huang, Kenji Watanabe, Takashi Taniguchi, Matthew R. Barnett, Ying Chen, Rodney S. Ruoff, and Lu Hua Li. Mechanical properties of atomically thin boron nitride and the role of interlayer interactions. Nature Communications, 8:1-9, 2017.

[10] Susanta Ghosh and Marino Arroyo. An atomistic-based foliation model for multilayer graphene materials and nanotubes. Journal of the Mechanics and Physics of Solids, 61(1):235-253, jan 2013.

[11] I. V. Grigorieva K. S. Novoselov, A. K. Geim, S. V. Morozov, D. Jiang, Y. Zhang, S. V. Dubonos and A. A. Firsov. Electric Field Effect in Atomically Thin Carbon Films. 306(5696):666-669, 2016 .

[12] Changgu Lee, Xiaoding Wei, Jeffrey W Kysar, and James Hone. of Monolayer Graphene. 321(July):385-388, 2008.

[13] Changgu Lee, Xiaoding Wei, Qunyang Li, Robert Carpick, Jeffrey W. Kysar, and James Hone. Elastic and frictional properties of graphene. Physica Status Solidi (B) Basic Research, 246(11$12): 2562-2567,2009$.

[14] Yu Ming Lin, Alberto Valdes-Garcia, Shu Jen Han, Damon B. Farmer, Inanc Meric, Yanning Sun, Yanqing Wu, Christos Dimitrakopoulos, Alfred Grill, Phaedon Avouris, and Keith A. Jenkins. Wafer-scale graphene integrated circuit. Science, 332(6035):1294-1297, 2011. 
[15] Kai Liu, Qimin Yan, Michelle Chen, Wen Fan, Yinghui Sun, Joonki Suh, Deyi Fu, Sangwook Lee, Jian Zhou, Sefaattin Tongay, Jie Ji, Jeffrey B. Neaton, and Junqiao Wu. Elastic properties of chemical-vapor-deposited monolayer MoS2, WS2, and their bilayer heterostructures. Nano Letters, 14(9):5097-5103, 2014.

[16] Ming Liu, Xiaobo Yin, Erick Ulin-Avila, Baisong Geng, Thomas Zentgraf, Long Ju, Feng Wang, and Xiang Zhang. A graphene-based broadband optical modulator. Nature, 474(7349):64-67, 2011.

[17] Piotr Matyba, Hisato Yamaguchi, Goki Eda, Manish Chhowalla, Ludvig Edman, and Nathaniel D. Robinson. Graphene and mobile ions: The key to all-plastic, solution-processed light-emitting devices. ACS Nano, 4(2):637-642, 2010.

[18] Vinh Phu Nguyen, Pierre Kerfriden, and Stéphane P.A. Bordas. Two- and three-dimensional isogeometric cohesive elements for composite delamination analysis. Composites Part B: Engineering, 60:193-212, 2014.

[19] K. S. Novoselov, V. I. Fal'Ko, L. Colombo, P. R. Gellert, M. G. Schwab, and K. Kim. A roadmap for graphene. Nature, 490(7419):192-200, 2012.

[20] Few-layer Black Phosphorus, Jin Tao, Wanfu Shen, Sen Wu, Lu Liu, Zhihong Feng, Chao Wang, Chunguang Hu, and Pei Yao. Mechanical and Electrical Anisotropy. ACS Nano, 9(Xx):1136211370, 2015.

[21] Ankit Rohatgi. Webplotdigitizer: Version 4.4, 2020.

[22] F. Schedin, A. K. Geim, S. V. Morozov, E. W. Hill, P. Blake, M. I. Katsnelson, and K. S. Novoselov. Detection of individual gas molecules adsorbed on graphene. Nature Materials, 6(9):652-655, 2007.

[23] Xiaoding Wei, Zhaoxu Meng, Luis Ruiz, Wenjie Xia, Changgu Lee, Jeffrey W. Kysar, James C. Hone, Sinan Keten, and Horacio D. Espinosa. Recoverable Slippage Mechanism in Multilayer Graphene Leads to Repeatable Energy Dissipation. ACS Nano, 10(2):1820-1828, 2016.

[24] Upendra Yadav, Shashank Pathrudkar, and Susanta Ghosh. Interpretable machine learning model for the deformation of multiwalled carbon nanotubes. Physical Review B, 103(3):1-7, 2021.

[25] Jianfeng Zang, Seunghwa Ryu, Nicola Pugno, Qiming Wang, Qing Tu, Markus J Buehler, and Xuanhe Zhao. Multifunctionality and control of the crumpling and unfolding of large-area graphene. Nature materials, 12(4):321-5, 2013.

[26] Kuan Zhang and Marino Arroyo. Understanding and strain-engineering wrinkle networks in supported graphene through simulations. Journal of the Mechanics and Physics of Solids, 2014.

[27] Kuang Zhang. Engineering patterns of wrinkles and bubbles in supported graphene through modeling and simulation. (September), 2015. 


\section{Copyright documentation}

1. Figure 1: "Figure 1. Membrane kinematics", by M. Arroyo and T. Belytschko., Finite element methods for the non-linear mechanics of crystalline sheets and nanotubes. International Journal for Numerical Methods in Engineering, 59(3):419-456, 2004. Downloaded from: https://onlinelibrary.wiley.com/doi/epdf/10.1002/nme.944

2. Figure 2: "The exponential map transforms the vector $\mathrm{w}=\mathrm{FA}$ tangent to the surface into a chord of the surface a.", by M. Arroyo and T. Belytschko., Finite element methods for the non-linear mechanics of crystalline sheets and nanotubes. International Journal for Numerical Methods in Engineering, 59(3):419-456, 2004. Downloaded from: https://onlinelibrary. wiley.com/doi/epdf/10.1002/nme.944

3. Figure 3: "Graphene honeycomb multi-lattice: the two simple Bravais lattices, depicted in different colors, are relatively displaced by the inner displacement $\mathrm{W}$, which consequently also affects the bond vectors which are transformed from $A_{0 i}$ into $A_{i}$. The unit cell of area $\mathrm{S} 0$ is also represented. It contains two nuclei and three in equivalent bonds.", by M. Arroyo and T. Belytschko., Finite element methods for the non-linear mechanics of crystalline sheets and nanotubes. International Journal for Numerical Methods in Engineering, 59(3):419-456, 2004. Downloaded from: https://onlinelibrary.wiley.com/doi/epdf/10.1002/nme.944

4. Figure 4: "Definition of domains used for integration in isogeometric analysis. Elements are defined in the parametric space as non-zero knot spans, $\left[\xi_{i}, \xi_{i+1}\right] \times\left[\eta_{j}, \eta_{j+1}\right]$ and elements in the physical space are images of their parametric counterparts." by Vinh Phu Nguyen, Isogeometric cohesive elements for two and three dimensional composite delamination analysis. Composites Part B: Engineering Volume 60, April 2014, Pages 193-212. Downloaded from: https://www. sciencedirect.com/science/article/pii/S1359836813007488?via\%3Dihub

5. Figure 7: "Schematic of doubly-clamped beam structure for 2D material before and after applying the indentation load" by Guoxin Cao and Huajian Gao, Mechanical properties characterization of two-dimensional materials via nanoindentation experiments. Progress in Materials Science, 103(June 2018):558-595, 2019. Downloaded from https://www. sciencedirect. com/science/article/pii/S0079642519300258

6. Figure 8: "Schematic of clamped circular thin plate model for 2D material under indentation load" by Guoxin Cao and Huajian Gao, Mechanical properties characterization of two-dimensional materials via nanoindentation experiments. Progress in Materials Science, 103(June 2018):558-595, 2019. Downloaded from https://www.sciencedirect.com/ science/article/pii/S0079642519300258

7. Figure 9: "Schematic of sandwich beam geometry under centrally pointed load", by Aleksey Falin et al., Mechanical properties of atomically thin boron nitride and the role of interlayer interactions Nature Communications, 8:1-9, 2017.Downloaded from https://www.nature. com/articles/ncomms 15815

8. Figure 13: "Optical microscopy image of a graphene film on a $\mathrm{SiO}_{2} / \mathrm{Si}$ substrate with microwells of $1.3 \mu \mathrm{m}$ in diameter;", "Load-displacement curves and the corresponding fittings.", by Aleksey Falin et al., Mechanical properties of atomically thin boron nitride and the role of interlayer interactions Nature Communications, 8:1-9, 2017.Downloaded from https://www. nature.com/articles/ncomms15815

9. Figure 14: "Image of suspended graphene membranes and AFM nanoindentation schematic.", "Load/deflection data for bilayer graphene" by Lee et al (2009)., Elasticand frictional properties of graphene.Physica Status Solidi (B) Basic Research, 246(11-12):2562-2567,2009. Downloaded from https://onlinelibrary.wiley.com/doi/full/10.1002/pssb.200982329 
10. Figure 21: "SEM images of different morphologies developed on the graphene sheet" by Jianfeng Zang et al., Multifunctionality and control of the crumpling and unfolding of largearea graphene.Naturematerials, 12(4):321-5, 2013. Downloaded from https://pubmed.ncbi. nlm.nih.gov/23334002/ 